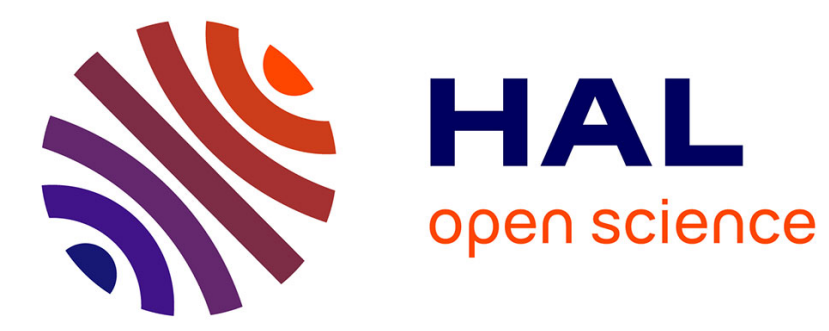

\title{
Large-scale streaky structures in turbulent jets
}

Petrônio Nogueira, André Cavalieri, Peter Jordan, Vincent Jaunet

\section{To cite this version:}

Petrônio Nogueira, André Cavalieri, Peter Jordan, Vincent Jaunet. Large-scale streaky structures in turbulent jets. Journal of Fluid Mechanics, 2019, 873, pp.211-237. 10.1017/jfm.2019.365 . hal02351588

\section{HAL Id: hal-02351588 \\ https://hal.science/hal-02351588}

Submitted on 6 Nov 2019

HAL is a multi-disciplinary open access archive for the deposit and dissemination of scientific research documents, whether they are published or not. The documents may come from teaching and research institutions in France or abroad, or from public or private research centers.
L'archive ouverte pluridisciplinaire HAL, est destinée au dépôt et à la diffusion de documents scientifiques de niveau recherche, publiés ou non, émanant des établissements d'enseignement et de recherche français ou étrangers, des laboratoires publics ou privés. 


\title{
Large-scale, streaky structures in turbulent jets
}

\author{
Petrônio A. S. Nogueira ${ }^{1} \dagger$, André V. G. Cavalieri ${ }^{1}$, Peter Jordan ${ }^{2}$ \\ and Vincent Jaunet ${ }^{2}$ \\ ${ }^{1}$ Divisão de Engenharia Aeronáutica, Instituto Tecnológico de Aeronáutica, São José dos \\ Campos, SP, Brazil \\ ${ }^{2}$ Département Fluides, Thermique, Combustion, Institut PPrime, CNRS-Université de \\ Poitiers-ENSMA, Poitiers, France
}

(Received $\mathrm{xx}$; revised $\mathrm{xx}$; accepted $\mathrm{xx}$ )

Streaks have been found to be an important part of wall turbulence dynamics. In this paper, we extend the analysis for unbounded shear flows, in particular a $M=0.4$ round jet, using measurements taken using dual-plane, time-resolved, stereoscopic particle image velocimetry taken at pairs of jet cross-sections, allowing the evaluation of the cross-spectral density of streamwise velocity fluctuations resolved into azimuthal Fourier modes. From the streamwise velocity results, two analyses are performed: the evaluation of wavenumber spectra (assuming Taylor's hypothesis for the streamwise coordinate) and a spectral proper orthogonal decomposition (SPOD) of the velocity field using PIV planes in several axial stations. The methods complement each other, leading to the conclusion that large-scale streaky structures are also present in turbulent jets where they experience large growth in the streamwise direction, energetic structures extending up to eight diameters from the nozzle exit. Leading SPOD modes highlight the largescale, streaky shape of the structures, whose aspect ratio (streamwise over azimuthal length) is about 15. The data was further analysed using SPOD, resolvent and transientgrowth analyses, good agreement being observed between the models and the leading SPOD mode for the wavenumbers considered. The models also indicate that the lift-up mechanism is active in turbulent jets, with streamwise vortices leading to streaks. The results show that large-scale streaks are a relevant part of the jet dynamics.

Key words: Authors should not enter keywords on the manuscript...

\section{Introduction}

The search for organised motion in high Reynolds-number turbulent flows has been an important object of study since the 1960s. The identification of order in flows previously considered to be entirely stochastic has broken old paradigms and established new ways of analysing turbulence. Study of the turbulent jet led to the discovery of axially extended, organised waves with amplitude modulation, referred to in the literature as wavepackets. Despite their low energy, such structures are found to be a central element of the jet dynamics, with amplitude growth related to the Kelvin-Helmholtz instability mechanism, and are important for sound generation on account of their spatiotemporal coherence (Mollo-Christensen 1967; Crow \& Champagne 1971; Crighton 1975; Cavalieri et al. 2013;

$\dagger$ Email address for correspondence: petronio@ita.br 
Jordan \& Colonius 2013; Breakey et al. 2017). In supersonic jets wavepackets generate sound through Mach-wave radiation (Tam \& Burton 1984; Sinha et al. 2014), but in subsonic jets sound radiation is associated with wavepacket jitter (Cavalieri et al. 2011; Cavalieri \& Agarwal 2014), which is postulated to be due to the interaction of wavepackets of low amplitude and azimuthal wavenumber with the energy-containing eddies of the turbulent field.

In wall-bounded flows, orderly structures have also been an object of study for some time, and in particular those characterised by streamwise vortices coupled with elongated streaks. Several such studies have been performed for boundary layers. Kline et al. (1967) identified organised streaky structures in the viscous sub-layer of boundary layers, with dynamics corresponding to a lift-up mechanism. Later, Smith \& Metzler (1983) explored some characteristics of these streaks, finding their spacing to be invariant with Reynolds number; the spanwise spacing is $\lambda_{z}^{+}=100$ for wall distances $y^{+}<5$; here, the + superscript indicates non-dimensional distances with respect to the friction velocity and the kinematic viscosity. Streaks located in the upper regions of the boundary layer $\left(y^{+}>10\right)$ have increasing spacing as the Reynolds number $R e$ is increased. The cited works motivated several research directions, especially concerning regions of the boundary layer responsible for turbulent kinetic energy production, and the role of streaks in this process (Smits et al. 2011). More recently, Hutchins \& Marusic (2007) performed PIV and hot-wire experiments and compared their results with those of a model, showing that the meandering motion of streaks can mask their real length: single-point statistics may lead to results that do not correspond to the reality. In their analysis, Hutchins \& Marusic found very large streaky structures in the logarithmic region of high-Reynolds number boundary layers, with streamwise extent larger than 20 times the boundary layer thickness; these were labelled as superstructures. Similar structures have also been found in turbulent channel and pipe flows by Monty et al. (2007).

The role of streaks and streamwise vortices in turbulence dynamics has been extensively studied for wall-bounded flows. Seminal work by Ellingsen \& Palm (1975) (later completed by Landahl (1980)) considered the stability of linearly sheared flow, reaching the conclusion that, though the flow may not support exponential growth of disturbances (in the modal linear stability analysis framework), there may nonetheless be significant disturbance amplification via a non-modal, linear mechanism. Streamwise vortices lead to a large amplification of the streamwise velocity, forming streaks, in what is referred to as the lift-up effect. This phenomenon was identified not only in Couette flow, but in other canonical wall-bounded flows such as plane Poiseuille flow (Gustavsson (1991)) and boundary layers (Hultgren \& Gustavsson (1981)). The main signature of this effect is the presence of streamwise elongated structures in the near-wall region of the turbulent flow. Work by Hamilton et al. (1995); Waleffe (1995), later developed by Schoppa \& Hussain (2002); Farrell \& Ioannou (2012), shows that these structures (streaks and streamwise vortices) are an intrinsic part of the dynamics of a wall-bounded turbulent shear flow, and constitute two of the building blocks that characterise the self-sustaining nature of turbulence. As summarised by Waleffe (1995), this process would consist of three main components: first, the occurence of (i) a spanwise modulation of the streamwise velocity by streamwise vortices, which generates streaks; followed by (ii) a breakdown of the streaks by an inflectional wavelike instability; finally, (iii) the streamwise vortices are regenerated by a non-linear self-interaction of the streak instability, restarting the process.

All of the studies discussed above consider wall-bounded shear flows. There are few studies of similar structures for unbounded, free-shear flows, such as shear layers and round jets. Bernal \& Roshko (1986) performed flow visualisation in water using a 
Schlieren technique in two perpendicular sections of a plane mixing layer, revealing the presence of Kelvin-Helmholtz vortices and streamwise streaks; such structures were generated in the upstream region of the flow and sufficiently amplified in the downstream region to be visible in the photographs. Later, Liepmann \& Gharib (1992) found similar structures in a transitional round jet of low Reynolds number ( $R e=5500$, based on the jet diameter) using particle image velocimetry (PIV) measurements. Their analysis concluded that streamwise vortices have a vital effect on the entrainment rate and in the dynamics of the shear flow, which agrees with wall-turbulence research, as stressed by Kozlov et al. (2002). Similarly, Jung et al. (2004) used 138 hot-wires to analyse the radial structure of the most energetic modes in a low-Mach-number jet, performing a slice-by-slice proper orthogonal decomposition at various axial stations. This was later complemented by the analysis of Tinney et al. (2008), who performed PIV measurements for a $M=0.85$ jet. In both studies, the authors found that the most energetic structures peak at non-zero azimuthal wavenumbers, with decreasing values of the peak wavenumber as the PIV plane or the hot-wires device were moved downstream. However, no clear association of such structure with the lift-up mechanism in wall-bounded flows was made,and a characterisation of the streamwise extent of these structures was still lacking.

Interest in the role played by streaks in free-shear flows has increased in recent years. Jiménez-González \& Brancher (2017) studied the problem of the disturbances that experience the largest temporal transient-growth in round jets, studying both static and diffusing base flows. The optimal initial disturbance was found to take the shape of streamwise vortices, leading to amplified streaks, with gains that scale with $R e^{2}$ regardless of the diffusive nature of the base flow. Moreover, the different azimuthal modes $m$ appear to be underpinned by different mechanisms: while $m \geqslant 2$ appears to be related to the lift-up mechanism (with growth rates that scale with $1 / m^{3}$ ), $m=1$ disturbances are more related to a shift-up mechanism, a dynamics that shifts the entire jet in the radial direction. Finally, Marant \& Cossu (2018) evaluated optimal transientgrowth in a hyperbolic-tangent mixing layer, reaching similar conclusions as JiménezGonzález \& Brancher (2017): the growth rate scales with $R e^{2}$ and the optimal initial disturbances consist of streamwise vortices, which give rise to streamwise streaks as they evolve in time. They also analysed how streaks can change the characteristics of the Kelvin-Helmholtz instability, finding both stabilising and destabilising effects depending on whether optimal sinuous or suboptimal varicose mode are considered in the analysis. Both studies are theoretical, and to date conclusive evidence of the presence of large-scale streaks in free turbulent shear flow is lacking. An identification of such structures would be of considerable interest, since their effect on Kelvin-Helmholtz wavepackets may aid in the modelling of turbulent jet noise.

The main objective of this paper is to show that streaky structures can also be found in unbounded, free-shear flows of high Reynolds number using the same tools used in wall-bounded turbulence, such as proper orthogonal decomposition, spatial Fourier transforms, resolvent and transient-growth analyses. We analyse a $M=0.4$ jet using PIV measurements and the cited methods. Results show a high resemblance with what is found for turbulent pipe flow and boundary layers, supporting the hypothesis that this structure is a fundamental component of turbulence dynamics, not only in wall-bounded flows, but in any shear flow.

The remainder of the paper is organised as follows. In $\S 2$ we present the experimental setup. In $\S 3$ we describe the spectral proper orthogonal decomposition (SPOD), resolvent and transient-growth analysis frameworks. Afterwards, in $\S 4$ we present the main results from the study, starting with a Fourier transform analysis, followed by the SPOD results 
and a comparison with the equivalent cases from resolvent and transient-growth analyses. The paper is closed with summary of the conclusions in $\S 5$.

\section{Experimental setup}

The present work uses the experimental database reported in Jaunet et al. (2017). All experiments were conducted at the "Bruit et Vent" jet-noise facility of the PPRIME Institute, Poitiers, France. The Mach number chosen for the analysis was $M=0.4$ and the nozzle diameter $D=0.05 \mathrm{~m}$, leading to a diameter-based Reynolds number of $R e=4.6 \times 10^{5}$; the corresponding momentum-thickness-based Reynolds number $R_{\theta}$ is $4 \times 10^{3}$. The settling chamber contains a series of honeycombs structures and grids to remove residual turbulence in the potential flow field. The flow is then accelerated into a convergent-straight nozzle, where the boundary layer is tripped to ensure transition to turbulence upstream the nozzle exit. Velocity measurements inside the nozzle confirm that the boundary layer is turbulent. Details on the exit flow condition can be found in Cavalieri et al. (2013) and Jaunet et al. (2017).

In order to decompose the field into azimuthal Fourier modes and build the crossspectral matrix, two time-resolved, stereoscopic particle image velocimetry (PIV) systems were used, which could be moved independently, as illustrated in figure 1 . The acquisition frequency of the systems was $5 \mathrm{kHz}(10 \mathrm{kHz}$ for each PIV system), leading to a sampling interval of $\Delta t U_{\text {jet }} / D=0.548$, where $U_{j e t}$ is the jet velocity. A total of 19414 image pairs was recorded for each acquisition point $\left(x_{1} ; x_{2}\right)$. The axial positions $x_{1}$ and $x_{2}$ of the PIV planes varied between $1 D \leqslant x_{1} \leqslant 8 D$ and $x_{1}<x_{2} \leqslant 8 D$ with a spacing of $\Delta x=0.5 D$; for each position, the first PIV system was kept fixed, while the second was moved downstream until it reached the end of the domain.

Both the jet flow and the surrounding air were seeded by injection of pure glycerine smoke particles in the pipe, upstream of the settling chamber. This ensures an homogeneous seeding with particles having diameters in the range of $1-2 \mu \mathrm{m}$, responding at frequencies up to $S t=f D / U_{\text {jet }}=20$, hence sufficiently small to follow the velocity fluctuations of interest in this study. The particle-image velocimetry calculations were carried out using a multipass iterative PIV algorithm (Scarano 2001). The first pass interrogation window size was set to $32 \times 32$ pixels and decreased to $16 \times 16$ pixels with an overlap of $50 \%$. This led to a resolution of one vector every $0.85 \mathrm{~mm}$ and PIV vector fields containing $140 \times 110$ velocity vectors. Outliers were detected using the universal outlier detection technique on $3 \times 3$ interrogation grids. We used a residual threshold of 2 , above which vectors were considered spurious (Westerweel \& Scarano 2005). Unexpectedly, no sign of peak-locking was observed in the data. It is believed that this is due to the fact that the particle images were dewarped (i.e. interpolated) prior to computation of the cross-correlation. This has a blurring effect on the particle images which confines the peak-locking effects beneath the PIV noise floor. In order to perform Fourier transform in the azimuthal direction, the instantaneous velocity fields were interpolated onto a polar grid of 32 points in the radial direction and 64 in azimuth for $r / D \leqslant 0.8$ using a bicubic interpolation. The spatial resolution of the interpolated field has been chosen to match the original one in order to avoid any loss of information. More details can be found in Jaunet et al. (2017).

In the following, we perform an analysis of the cross-spectral density (CSD) matrix of the PIV data. The CSD is obtained using the Welch periodogram technique with blocks of $n_{f f t}=128$ samples, with overlap of $50 \%$. The minimum resolved frequency, and therefore the frequency resolution of the spectral content, is $\Delta S t=0.015$. A standard Hann tapering window was applied to all blocks of data to minimise spectral leakage. 

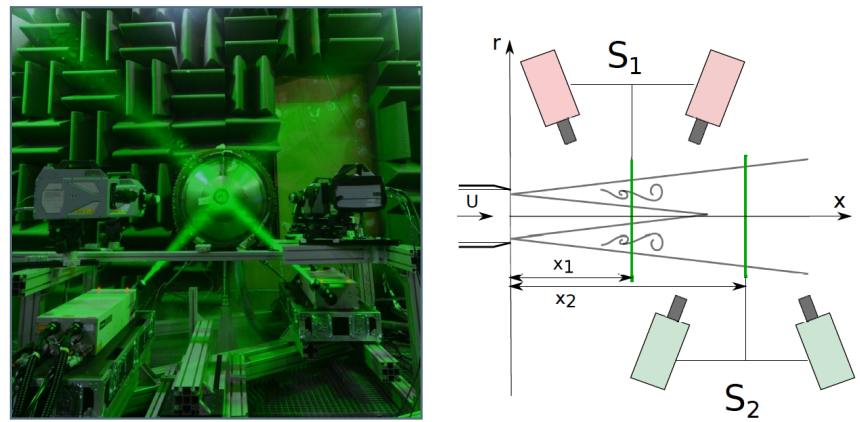

FiguRE 1. Experimental setup (left) and coordinate system adopted by the PIV planes (right). Taken from Jaunet et al. (2017).

\section{Analysis and modelling methods}

The analysis and modelling methods employed are described in this section. These involve spectral proper orthogonal decomposition, resolvent analysis and transient-growth analysis.

\subsection{Spectral Proper Orthogonal Decomposition}

The experiment as it was performed allows us to build a cross-spectral matrix for the streamwise velocity component. The data is decomposed into mean and fluctuating components:

$$
\tilde{u}(\mathbf{x}, t)=U(\mathbf{x}, r)+u^{\prime}(\mathbf{x}, t),
$$

where $\mathbf{x}=(x, r, \theta)$, with the mean taken in time. The fluctuations are then Fourier transformed in time and in azimuth so that:

$$
u^{\prime}(\mathbf{x}, t)=\int_{\omega} \sum_{m} u(x, r, m, \omega) \mathrm{e}^{\mathrm{i} m \theta} \mathrm{e}^{-\mathrm{i} \omega t} \mathrm{~d} \omega,
$$

where $m$ is the azimuthal wavenumber and $\omega$ is the frequency.

Spectral proper orthogonal decomposition (SPOD) is used to identify the most energetic coherent structures. Following Jung et al. (2004); Cavalieri et al. (2013), we can write the SPOD integral equation as:

$$
\int_{\Omega} R\left(x, x^{\prime}, r, r^{\prime}, m, \omega\right) \xi\left(x^{\prime}, r^{\prime}, m, \omega\right) r^{\prime} d r^{\prime} d x^{\prime}=\sigma(m, \omega) \xi(x, r, m, \omega),
$$

where $R$ is the two-point cross-spectral tensor of the streamwise velocity fluctuations, $\sigma$ is the eigenvalue and $\xi$ is the corresponding eigenfunction. The integral is evaluated in the whole experimental domain $\Omega$.

The eigenvalue problem of eq. 3.3 leads to real, positive eigenvalues $\sigma$ and orthonormal eigenfunctions $\xi$. The expression as written in equation 3.3 relates structures extended over the whole domain, given by the eigenfunctions $\xi$, with their respective energies $\sigma$, allowing us to find the most energetic structures for each $m-\omega$ pair. The dual PIV configuration used in the experiments only allowed accurate measurement of the streamwise component $u$; thus, the energy represented by the SPOD modes refers solely to the $u$ component. Compared to previous works (Jung et al. 2004; Tinney et al. 2008; 
Cavalieri et al. 2013), which applied POD separately for a number of jet slices, the present spectral POD modes are defined over the entire domain, thanks to the use of two time-resolved PIV planes to obtain the azimuthally-resolved cross-spectral density.

\subsection{Resolvent analysis}

Resolvent analysis has been widely used in several kinds of flow, and is a useful tool to analyse both laminar (focusing on identifying mechanisms of transition) and turbulent flows, which is the present case. We follow the approach described by McKeon \& Sharma (2010), using the resolvent to identify optimal response structures in the jet and the shape of the associated optimal forcing. In a locally parallel framework, we consider Fourier transformed variables in the streamwise direction in addition to the Fourier series in azimuthal wavenumber $m$ and use the mean flow $U\left(x_{1}, r\right)$ at a given $x_{1}$ station as base flow. The Linearised Navier-Stokes equations in the input-output form can be written as:

$$
\begin{gathered}
\frac{\partial}{\partial t}\left(\mathscr{H}\left[\begin{array}{l}
\phi \\
p
\end{array}\right]\right)\left(k_{x}, r, m, t\right)=\mathscr{L}\left[\begin{array}{l}
\phi \\
p
\end{array}\right]\left(k_{x}, r, m, t\right)+\left[\begin{array}{l}
\mathbf{f} \\
0
\end{array}\right]\left(k_{x}, r, m, t\right), \\
\phi_{u}\left(k_{x}, r, m, t\right)=\mathscr{C}\left[\begin{array}{l}
\phi \\
p
\end{array}\right]\left(k_{x}, r, m, t\right),
\end{gathered}
$$

where $\phi=\left[\begin{array}{lll}u & v & w\end{array}\right]^{T}$ contains the velocity components (streamwise, radial and azimuthal, respectively) and $\mathbf{f}=\left[\begin{array}{lll}f_{x} & f_{r} & f_{\theta}\end{array}\right]^{T}$ is a forcing term (which is considered to gather the nonlinear terms in the momentum equation); the continuity equation is linear and thus has no forcing term. The matrix $\mathscr{L}$ defines the evolution of the disturbances and $\mathscr{C}$ is a filter matrix that isolates only the streamwise component of the velocity in the vector $\phi_{u}=$ $\left[\begin{array}{lll}u & 0 & 0\end{array}\right]^{T}$ for consistency with the SPOD results, which account only for the amplitude of the streamwise component; however, nearly no difference is found in the results if all velocity components are considered in the analysis, since the flow response is dominated by the streamwise component. All the matrix operators are described in Appendix A. The radial discretisation is done using Chebyshev polynomials in $r$, following Trefethen (2000), and in order to diminish the computational cost, we adopt the same mapping of the radial domain used by Lesshafft \& Huerre (2007) to concentrate Chebyshev nodes at the shear layer and potential core of the jet; boundary conditions for the azimuthal wavenumbers were implemented in the same fashion as the cited work. The base flow was chosen to be a slice of the experimental mean flow $U\left(x_{1}, r\right)$ at $x_{1} / D=2$, but the choice of other streamwise stations $x_{1}$ was seen to lead to similar results. If the linear operator $\mathscr{L}$ is stable one can define a frequency response function between input and output. Assuming Fourier-transformed variables in time, as in equation 3.2, one can write:

$$
\begin{array}{r}
{[-\mathrm{i} \omega \mathscr{H}-\mathscr{L}]\left[\begin{array}{l}
\phi \\
p
\end{array}\right]=\left[\begin{array}{l}
\mathbf{f} \\
0
\end{array}\right]} \\
\Rightarrow\left[\begin{array}{l}
\phi \\
p
\end{array}\right]=[-\mathrm{i} \omega \mathscr{H}-\mathscr{L}]^{-1}\left[\begin{array}{l}
\mathbf{f} \\
0
\end{array}\right] .
\end{array}
$$

Adopting a compact form for the forcing term via the matrix operator $\mathscr{B}$, shown in the Appendix, the previous equation leads us to

$$
\phi_{u}=\mathscr{C}[-\mathrm{i} \omega \mathscr{H}-\mathscr{L}]^{-1} \mathscr{B} \mathbf{f}=\mathscr{R} \mathbf{f},
$$


where the resolvent operator is given by $\mathscr{R}=\mathscr{C}(-\mathrm{i} \omega \mathscr{H}-\mathscr{L})^{-1} \mathscr{B}$. Finding the optimal forcing and associated response of the problem involves solving the following problem:

$$
s_{1}^{2}=\max _{f}\left\{\frac{\langle\mathscr{R} \mathbf{f}, \mathscr{R} \mathbf{f}\rangle_{\phi_{u}}}{\langle\mathbf{f}, \mathbf{f}\rangle_{f}}\right\},
$$

which can be achieved by performing a singular value decomposition of the operator $\mathscr{R}$. Usually, the inner products chosen in equation 3.9 are defined according to energy norms for input $\mathbf{f}$ and output $\phi_{u}$. In the present work we choose a norm $\langle\cdot, \cdot\rangle_{\phi_{u}}$ that weights the problem so as to localise responses inside $(r / D<1)$ in order to avoid free-stream modes, as reported by Dergham et al. (2013); using that formulation, we perform the analysis for a weighted resolvent operator, isolating the optimal and suboptimals for each frequency and wavenumbers leading to amplified responses in the jet region. The weighting function $\mathbf{W}_{\phi_{u}}$ is given as

$$
\mathbf{W}_{\phi_{u}}=\operatorname{diag}\left[0.5(1-b)\left(1+\tanh \left(r_{p} / D-r / D\right)\right)+b\right] \mathbf{W}_{c h e b},
$$

where $b$ is a small parameter used to avoid singularities in the matrix inversion and other problems with the Cholesky factorisation; $r_{p} / D=1$ is the position where the function goes from 1 to 0 , and $\mathbf{W}_{c h e b}$ are the standard quadrature weights for Chebyshev polynomials. With this choice of parameters, we can avoid modes related to the excitation of free-stream disturbances and focus on the structures close to the shear region of the jet. The inner products in eq. 3.9 become, in matrix form, $\langle\mathscr{R} \mathbf{f}, \mathscr{R} \mathbf{f}\rangle_{\phi_{u}}=\mathbf{f}^{H} \mathscr{R}^{H} \mathbf{W}_{\phi_{u}} \mathscr{R} \mathbf{f}$ and $\langle\mathbf{f}, \mathbf{f}\rangle_{f}=\mathbf{f}^{H} \mathbf{W}_{f} \mathbf{f}$, and the resolvent modes are obtained by taking the SVD of $\mathbf{W}_{\phi_{u}}^{\frac{1}{2}} \mathscr{R} \mathbf{W}_{f}^{\frac{1}{2}}$, as in Tissot et al. (2017); Towne et al. (2018). For the present problem, we choose $\mathbf{W}_{f}=\mathbf{W}_{\text {cheb }}$.

With the defined formulation, the output of the resolvent analysis is the optimal forcing that would maximise streamwise velocity in regions where the turbulence intensity is higher $(r / D<1)$. The most amplified response corresponds to turbulent structures most likely to be excited by non-linear dynamics (Cavalieri et al. 2019). Moreover, if non-linear terms in the Navier-Stokes system are modelled as white noise, the resulting SPOD modes should be equal to response modes from resolvent analysis Towne et al. (2018).

\subsection{Transient growth}

While the resolvent analysis obtains the harmonic forcing that generates the maximum response, transient growth shifts the analysis to the time domain. It focuses on finding the initial disturbance that will experience the largest growth in time, in a linear nonmodal mechanism. Studies like Hanifi et al. (1996); Butler \& Farrell (1992); Del Alamo \& Jimenez (2006); Pujals et al. (2009); Cossu et al. (2009) highlight the importance of such analysis for both laminar and turbulent flows. Indications of the optimal paths for transition to turbulence and even the optimal structures present in turbulent flows can be found, and the latter is the focus of the present work (with optimal meaning the structures related to the maximum amplification, which are thus more likely to be found in the experimental data). Considering the problem without forcing (i.e. neglecting non-linear terms), we can write the evolution of disturbances in the flow as:

$$
\frac{\partial}{\partial t}\left(\mathscr{H}\left[\begin{array}{l}
\phi \\
p
\end{array}\right]\right)\left(k_{x}, r, m, t\right)=\mathscr{L}\left[\begin{array}{l}
\phi \\
p
\end{array}\right]\left(k_{x}, r, m, t\right),
$$

an initial value problem with the solution 


$$
\left[\begin{array}{l}
\phi \\
p
\end{array}\right]\left(k_{x}, r, m, t\right)=\mathrm{e}^{\mathscr{L} t}\left[\begin{array}{l}
\phi \\
p
\end{array}\right]\left(k_{x}, r, m, 0\right)
$$

Hence, the optimal growth curve is given by:

$$
G(t)=\max _{\phi(0) \neq 0} \frac{\|\phi(t)\|}{\|\phi(0)\|},
$$

disregarding the pressure term in the energy norm calculation; || || refers to the euclidean norm. The time in the growth curves is normalised by $D / U_{x}$, where $U_{x}$ is the peak of the mean velocity at the analised axial station. The equation above is then solved using the eigenfunction expansion method described in Schmid \& Henningson (2012), considering nearly all eigenfunctions; only those with spurious eigenvalues were discarded. The structure of the response at the time of optimal growth can be identified by this computation, as well as the initial disturbance that generates that field. Also, since the solution involves a singular value decomposition, the suboptimals at a given time can also be obtained in the same calculation. Note that no turbulent viscosity was added to the model for both resolvent and transient growth analyses.

\section{Results}

The results are divided into two distinct parts. In the first, we treat each cross-section of the jet separately, transforming the time variable $t$ into a pseudo-axial coordinate $x_{t}$ using Taylor's hypothesis, as in Marusic et al. (2017). Moreover, the azimuthal coordinate $\theta$ is shown as a pseudo span $z=r \theta$. We obtain thus $\left(x_{t}, z\right)$ fields, which are Fourier transformed and compared to results of boundary layer experiments so as to evaluate similarities between structures in a turbulent jet and the large-scale, elongated structures seen in turbulent boundary layers. In the second analysis, we use all the pairs $\left(x_{1}, x_{2}\right)$ of the experiments to build the cross spectral matrix and obtain the SPOD modes for each azimuthal mode, leading to a further characterisation of the jet structures.

We then analyse the radial shapes of these structures. Slices of the SPOD modes are compared with the equivalent resolvent response modes at the same frequency and azimuthal wavenumber. The same results are then compared with transient growth responses at the time of optimal growth. In both analyses, the optimal forcing and optimal initial disturbances are analysed, relating the phenomena present in the turbulent jet with those found in wall-bounded turbulent flows.

\subsection{Velocity fluctuations studied with Taylor's hypothesis}

Three representative PIV planes $(x / D=3,5,7)$ are selected, and the time coordinate transformed using Taylor hypothesis, $x_{t}=t U_{\text {ref }}$, where $U_{\text {ref }}$ is chosen to be the mean velocity at the radial station analysed $(r / D=0.55)$. A pseudo-spanwise coordinate $z$ is obtained by rescaling azimuth $\theta$ as $z=r \theta$. Axial velocity fluctuations at the planes $\left(x_{t}, z\right)$ can be seen in Figure 2.

Looking closely at figure 2, one can identify elongated streaky structures, resembling those found in wall-bounded flows, such as boundary layers (Hutchins \& Marusic 2007), pipes (Hellström et al. 2011) and channel flow (Monty et al. 2007), usually associated with very large scale motions or superstructures in wall-bounded turbulence studies (Smits et al. 2011). As is the case in turbulent boundary layers superstructures (Hutchins \& Marusic 2007), the streaks meander in the axial direction in this free shear flow. Also, 


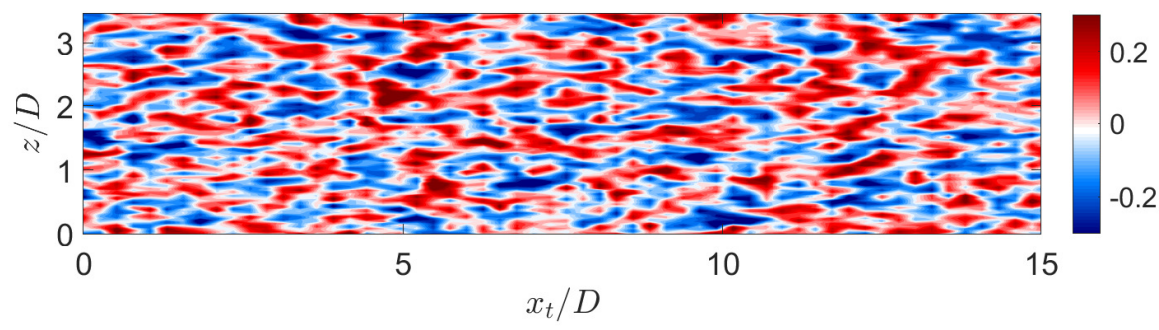

(a) $x / D=3$

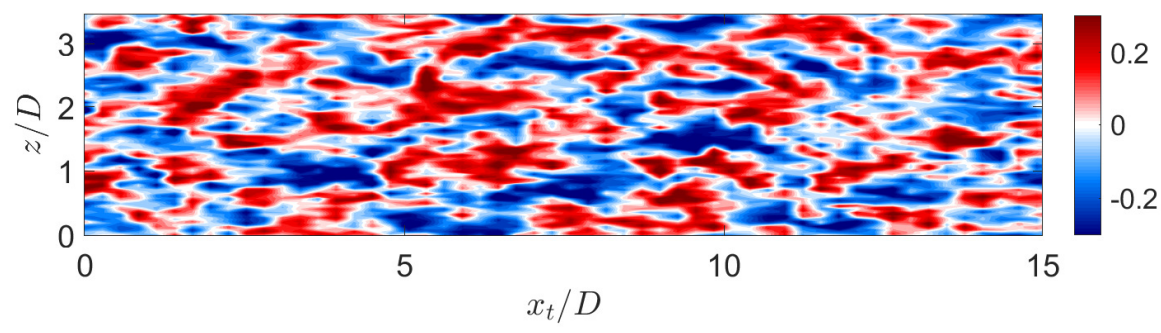

(b) $x / D=5$

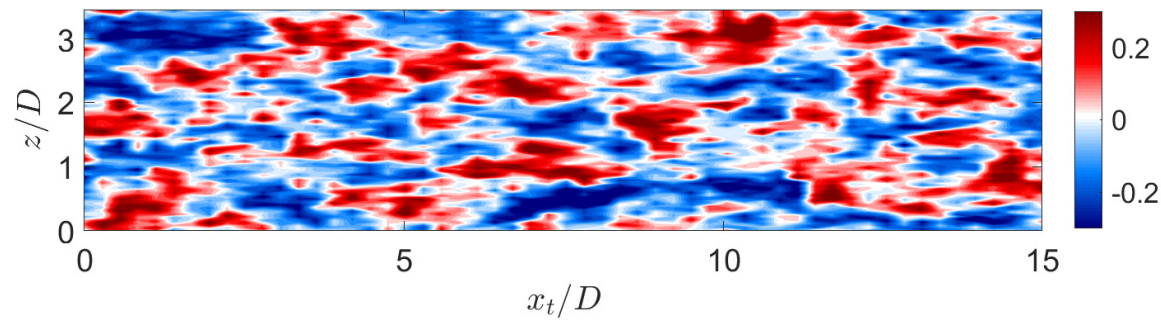

(c) $x / D=7$

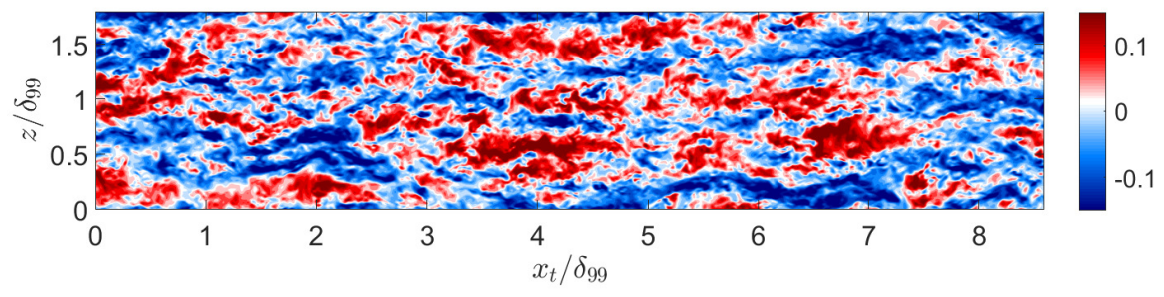

(d) Turbulent Boundary Layer $\left(y^{+}=120\right)$

FiguRE 2. Axial velocity fluctuations over a $\left(x_{t}, z\right)$ plane for a $M=0.4$ jet for several axial stations $(x / D=3,5,7$, respectively) and $r / D=0.55$ (a,b,c). The data was normalised by the mean streamwise velocity at the given radial station. Colour ranges from -0.3 to 0.3.Axial velocity fluctuations of large-eddy simulation results of a turbulent boundary layer at $y^{+}=120$ and $\operatorname{Re}_{\delta_{\theta}}=4430$ (Eitel-Amor et al. 2014) are also shown (d).

the pseudo-spanwise wavelength changes from small values at positions near the nozzle, to larger values as the PIV measurement station is moved downstream; the streak width for $x / D=3$ is smaller than for the two other positions considered. This behaviour can be expected since, moving downstream, the shear-layer thickness also increases; the scaling 
of streak sizes with the local thickness is explored next. To highlight the resemblance of these streaks with the large scale structures from turbulent boundary layers, we show the results from a large-eddy simulation performed by Eitel-Amor et al. (2014) at $y^{+}=120$ and $\operatorname{Re}_{\delta_{\theta}}=4430$ in figure 2(d). Visually, these structures, that scale with the boundary layer thickness $\left(\delta_{99}\right)$, show high similarity with the ones shown here for turbulent jets.

From the fields depicted in figure 2, a spatial Fourier transform is performed in both pseudo-axial and spanwise directions for $r / D=0.42$ and $r / D=0.55$, using the mean velocity at each radial station as the reference velocity for Taylor's hypothesis. The wavenumber spectra are shown in figure 3 , from which some characteristics of the flow can be educed.

The first is that, for $r / D=0.42$, the peak wavenumber is always located at $k_{x} \neq 0$ : it is already known that organised structures in the shape of axially extended wavepackets, with spatial evolution characterised by the Kelvin-Helmholtz instability, are present in this kind of flow (Cavalieri et al. 2013; Jordan \& Colonius 2013). Nevertheless, considerable energy is also located at $k_{z} \neq 0$, which constitutes the signature of the streaky structures described above. As the streamwise position of the PIV plane is moved downstream, the energy spectrum becomes progressively concentrated around a specific spanwise wavenumber $\left(k_{z} \delta_{\theta} \approx 1\right)$. The main cause of this change in the wavenumber peak is the growth and decay mechanism of wavepackets, the structures related to $k_{x} \neq 0$ and low $k_{z}$ : for regions near the nozzle, these structures have high amplitudes due to the high growth rate of the Kelvin-Helmholtz mode in regions of higher shear. These structures saturate and decay further downstream, causing their energy to decrease. On the other hand, the energy of the structures related to $k_{x}=0$ seem to grow as we move downstream. One important fact is that there is significant energy for $k_{x} \rightarrow 0$ and $k_{z} \delta_{\theta} \approx 1$ for all positions considered. Figures $3(\mathrm{~b}, \mathrm{~d}, \mathrm{f})$ show the same spectrum for $r / D=0.55$, a region where wavepackets have lower amplitudes; one can see that the peak wavenumber in the spanwise direction remains around $k_{z} \delta_{\theta} \approx 1$, but now for $k_{x}=0$. This is a sign that this region is not dominated by the wavepacket dynamics, but by the streamwise elongated structures seen in figure 2 . Also, this result relates to what is visually perceived in figure 2: streaky structures have wavenumbers scaling with the local momentum thickness, and thus when plotted as a function of jet diameter one observes larger streaks at downstream stations.

To further highlight the relationship of these structures to those found in wall-bounded flows, we compare, in figure 3, the wavenumber spectrum of this flow with that of the turbulent boundary layer studied by Tomkins \& Adrian (2005). Even though the Reynolds number of the experiments differs $\left(R e_{\theta}=92542\right.$ at $x / D=7$ for the present experiment and $R e_{\theta}=1015$ for the boundary layer), the peak position in the low $k_{x}$ region is very similar for both cases (around $k_{z} \delta_{\theta}=1$ ), confirming that the structures found experimentally in the present case are similar to those that are ubiquitous in boundary layers, having even a comparable energy spectrum. Previous work (Bogey et al. 2011; Cavalieri et al. 2013) has highlighted this resemblance in the energy distribution via azimuthal Fourier-mode decomposition, showing similar peaks in the wavenumber spectrum with the proper scaling.

Finally, the premultiplied spectra for several axial positions of the PIV measurement, $k_{x}=0$ and $r / D=0.55$ can be seen in Figure 4(a). The energy amplitudes of the streaks increase monotonically as we move the plane downstream, giving evidence that these structures are present with a higher amplitude in regions even further away from the nozzle, always peaking at the same value of $k_{z} \delta_{\theta}$. The behaviour of the peak energy with the streamwise position of the PIV plane is shown in figure 4(b). 


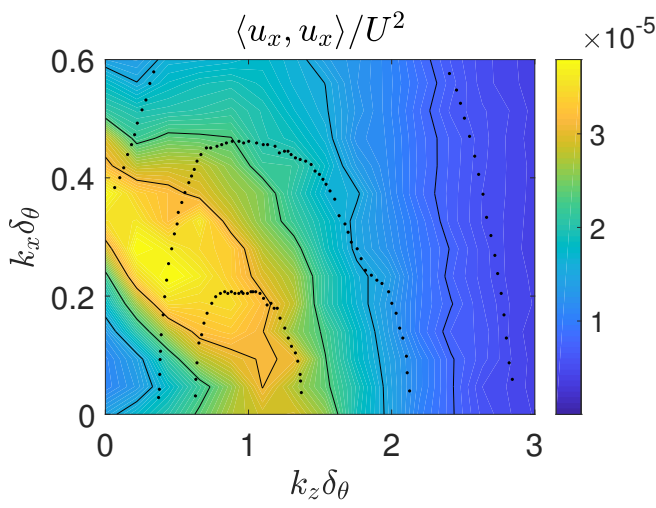

(a) $x / D=3, r / D=0.42$

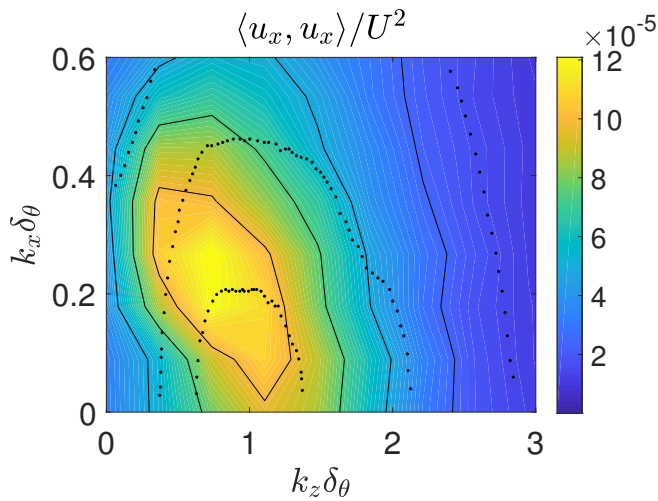

(c) $x / D=5, r / D=0.42$

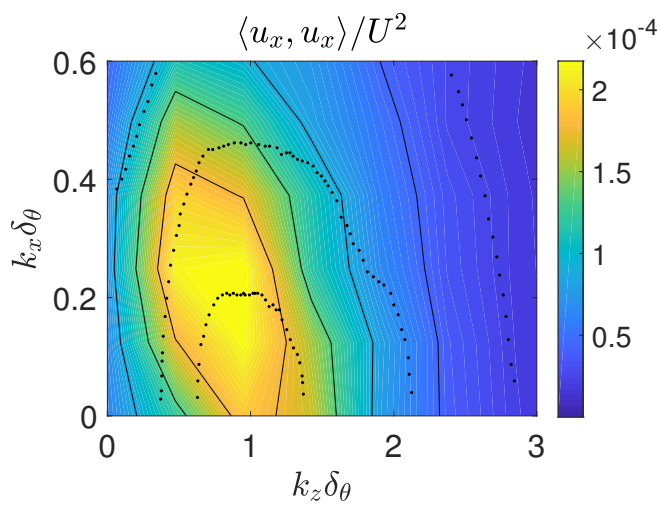

(e) $x / D=7, r / D=0.42$

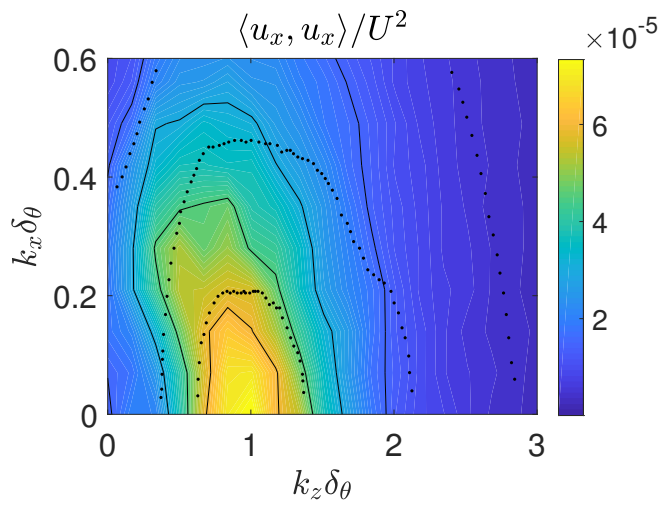

(b) $x / D=3, r / D=0.55$

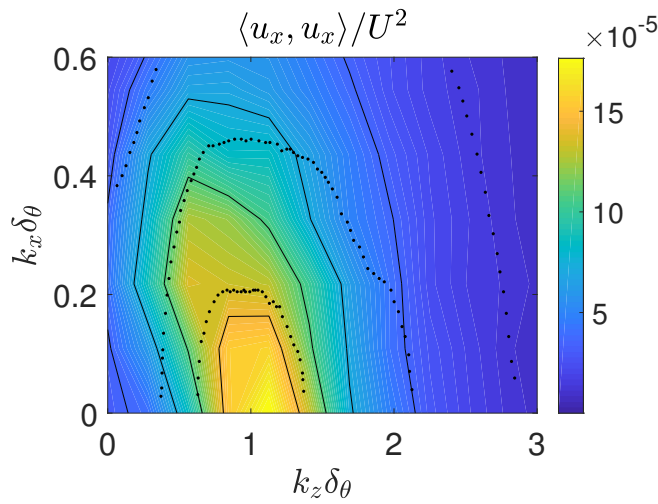

(d) $x / D=5, r / D=0.55$

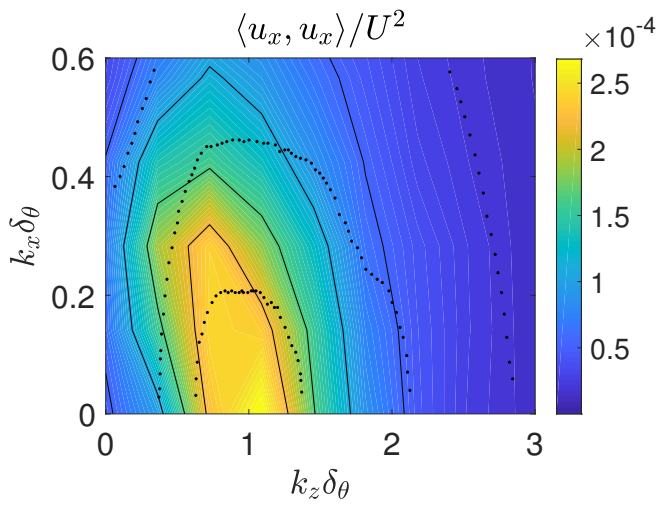

(f) $x / D=7, r / D=0.55$

FiguRE 3. Energy spectrum (colours, full contours) in the $\left(k_{x}, k_{z}\right)$ domain for a $M=0.4$ jet for several axial stations $(x / D=3,5,7$, respectively) and $r / D=0.42,0.55$. A comparison with results from Tomkins \& Adrian (2005) is also shown (dotted contours). Spectrum axes are normalised by the momentum thickness $\delta_{\theta}$ 


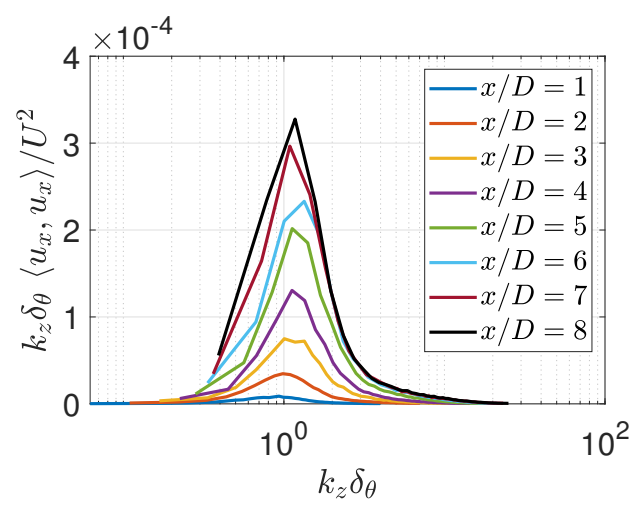

(a) Premultiplied energy spectra

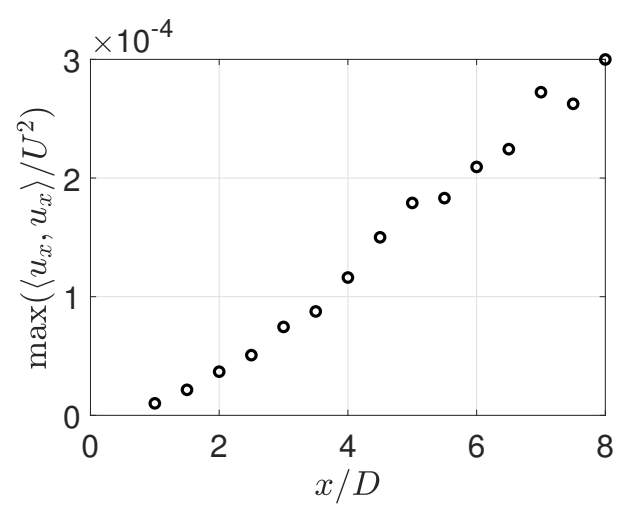

(b) Maximum energy as a function of $x / D$

FiguRE 4. Spectra for $k_{x}=0$ and $r / D=0.55$.

\subsection{Spectral Proper Orthogonal Decomposition}

Solving the eigenvalue problem defined by equation 3.3 using standard methods, we can obtain the energy of each SPOD mode $\sigma_{n}(m, \omega)$ and the respective eigenfunction $\xi^{(n)}(x, r, m, \omega)$. Figure 5(a) shows the leading SPOD-mode energies for several Strouhal numbers $S t=\omega D /\left(2 \pi U_{\text {jet }}\right)$ and azimuthal modes $m$. The high-energy region for $m=0$ was shown to be related to a coherent wavepacket structure, as analysed by Semeraro et al. (2016); Lesshafft et al. (2018), and is not the focus of the present work. Instead, we consider the $m>1$ region of the spectrum, where the reported streaky structures occur; in this region, the highest energies are found for $S t=0$ (except for $m=1$ and 2), meaning that the structures are nearly steady in time, being related to a slow dynamic of the flow; here, SPOD modes for $S t=0$ should be interpreted as the $S t \rightarrow 0$ limit, with structures whose time scale is larger than the segment size used in Welch's method. Even for $m=1$ and 2 the peak still occurs at low Strouhal numbers, supporting the hypothesis of a single mechanism. The energies of the first 4 SPOD modes for several azimuthal wavenumbers $m$ and $\omega=0$ can be seen in Figure 5(b). As can be seen, a peak in $m=3$ arises for $n=1$ (which matches with the POD analysis performed by Hellström et al. (2015) for turbulent pipe flow) with a substantial separation between mode 1 and the suboptimals at the low $m$ region.

Following the analysis performed by Hellström et al. (2016), we define the azimuthal wavelength as $\lambda_{\theta}=2 \pi / m$, which leads to a characteristic length of $\lambda_{\theta} R$, where $R=$ $D / 2$. To obtain the characteristic streamwise development of the first SPOD mode, the eigenfunctions were radially integrated $\left(\Xi(x)=\int\left|\xi^{(1)}\right| r d r\right)$ and then the axial coordinate was normalised using this new characteristic length. Normalising also the amplitude of each mode by its maximum, we find a collapse for these radially integrated first SPOD modes for azimuthal wavenumbers $m \geqslant 3$. This underlines the fact that $\lambda_{\theta}$ is the important length for the most energetic structures in this flow, highlighting the similarity with what is found for turbulent pipe flow in Hellström et al. (2016). However, the present analysis differs from the cited work mainly in the direction where this similarity was found: Hellström et al. (2016) found the disturbances in pipe flow to be self-similar in the radial direction, whereas we found a similarity and a characteristic length in the streamwise direction.

A striking feature of Figure 6(a) is that the leading modes are very large scale motions, extending several jet diameters in $x$. For instance, $m=10$ structures have length of 


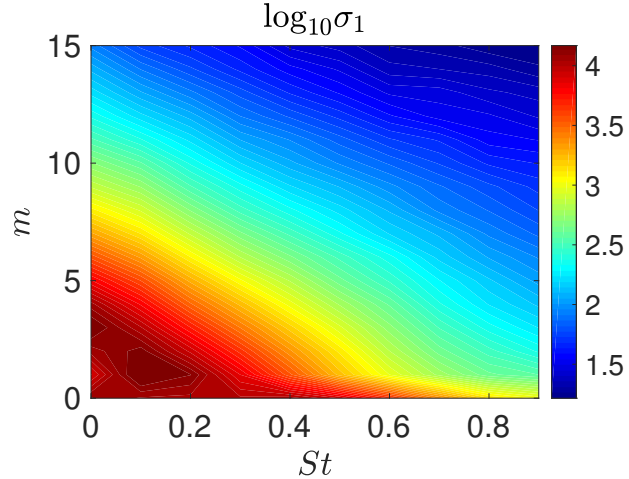

(a) First SPOD-mode energy

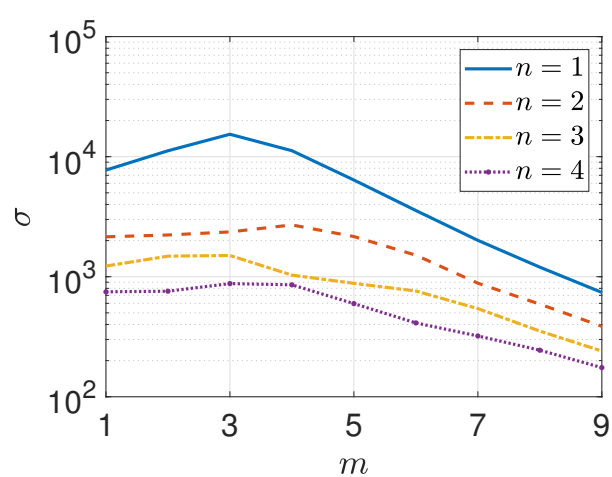

(b) SPOD-mode energies for $\omega=0$

FIGURE 5. First SPOD energy for several Strouhal numbers and azimuthal modes (a) and SPOD energies for each azimuthal mode for $\omega=0$ (b).

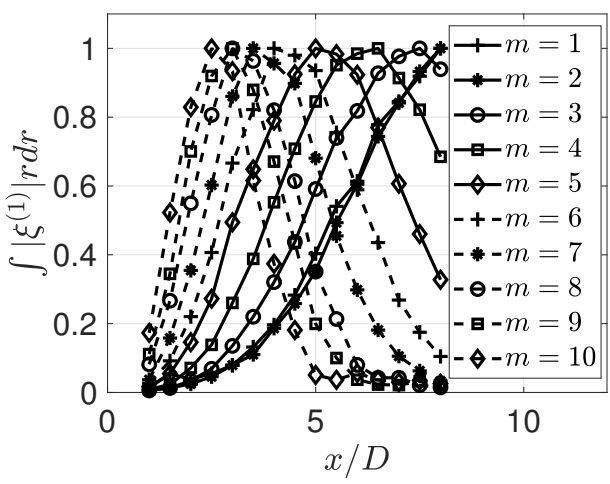

(a) Axial coordinate normalised by $D$

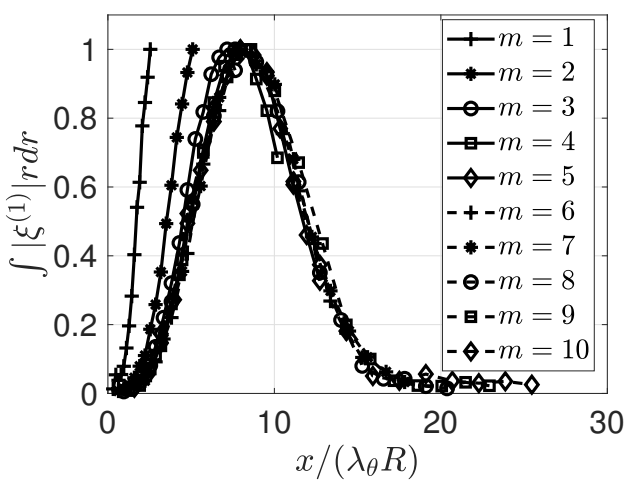

(b) Axial coordinate normalised by $\lambda_{\theta} R$

FIGURE 6. Shape of the first SPOD mode for several azimuthal modes.

about 5 jet diameters, and lower- $m$ modes are even larger than that. The aspect ratio of structures can be estimated through the collapse found in Figure 6(b), and corresponds, for $m \geqslant 3$, to a ratio of axial over azimuthal length equal to about 15, highlighting again the streaky shape of these modes.

The shapes of the first and second SPOD modes for $S t=0$ and several azimuthal wavenumbers can be seen in Figure 7 and 8. The main behaviour is similar for all modes: the structures start to grow from the near-nozzle region, at radial locations close to the shear layer, where linear mechanisms are expected to be relevant, and experience a substantial growth in space, with high amplitudes in different regions of the flow. As a consequence, we can identify the regions where each azimuthal mode is acting more effectively. For instance, $m=1$ and 2 are related to disturbances more concentrated at regions with $r / D<0.5$ and higher $x / D$. The oscillations in the streamwise direction of the second SPOD mode for higher azimuthal wavenumbers, shown in figure 8, also support the idea that these modes are related to streaky structures, representing their meandering motion observed in figure 2 by the phase opposition between upstream and downstream positions. One can see that the first and second SPOD modes do not decay within the region covered by the measurements for $m=1 ; 2$. Figure 7 also shows that 

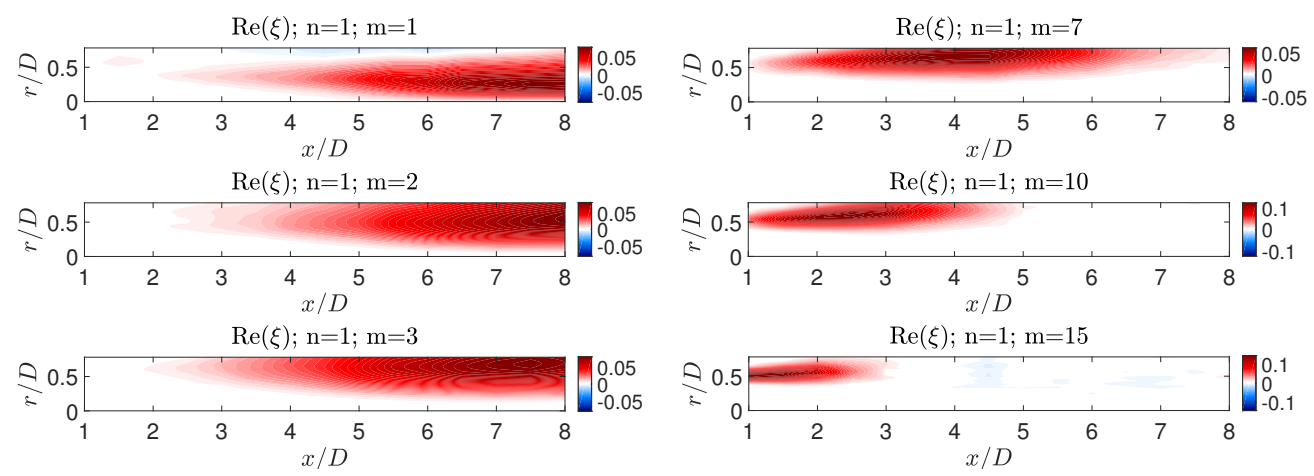

FiguRE 7. Streamwise velocity fluctuations of the first SPOD mode for several azimuthal modes.
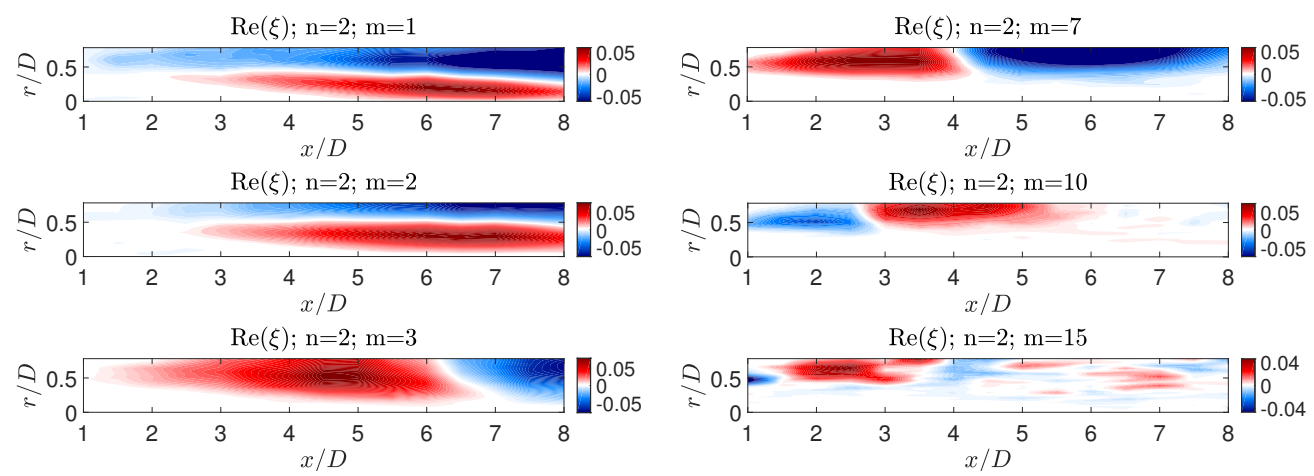

FiguRE 8. Streamwise velocity fluctuations of the second SPOD mode for several azimuthal modes.

all modes are located close to the nozzle as we increase the azimuthal wavenumber; this information, combined with the fact that streaks have higher amplitudes at downstream positions, which was inferred from figure 4(a), explains what is seen in the energy distribution of the SPOD modes: since lower azimuthal modes are related to more elongated structures, modes related to lower $m$ should be more energetic than the others at downstream positions.

On the other hand, for $m \geqslant 3$, the modes have higher amplitudes close to the lipline, highlighting their action in the region of maximum shear. The axial support of the modes decreases with increasing $m$, following the decrease of $\lambda_{\theta}$, as seen in Figure 6 , approaching progressively the nozzle. The fact that the peak position of these high azimuthal wavenumber streaks is in the upstream region indicates that these modes may well be captured by linear analysis. Particularly, the first SPOD mode for $m=10$ has a high amplitude at $x / D=2$; figure 9 shows a sketch allowing this mode to be visualised with an instantaneous field at that position. It can be seen that the flow also possesses the oscillatory behaviour in azimuth, displaying an instantaneous cross-section of the streaky structures at that position with the correct radial support. Looking at lower radial positions, the signature of an $m=1$ streaky structure can also be identified, albeit with smaller amplitudes, which again agrees with the analysis of figure 7 , since low- $m$ modes are more relevant for higher $x / D$. The similarities between the structure of this mode and the instantaneous velocity field are clear, showing that these streaks are indeed present in snapshots of the turbulent jet. 


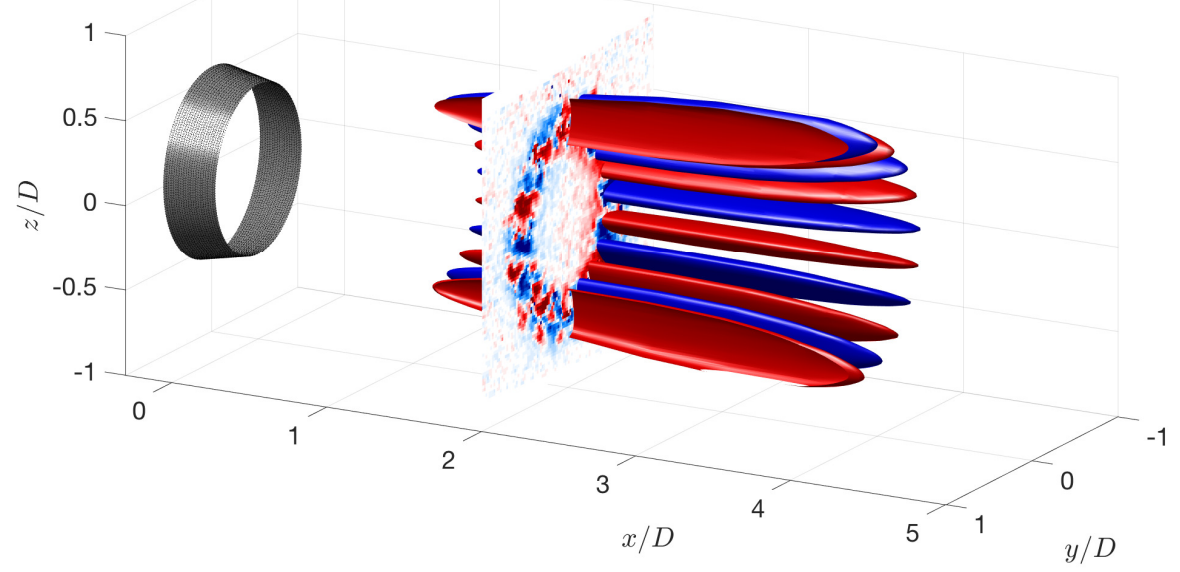

Figure 9. Isosurfaces of $40 \%$ of the maximum amplitude of the first SPOD mode (positive values in red and negative ones in blue) and $m=10$; contours rended for $0<\phi<\pi$ for clarity. An instantaneous field from the experiments at $x / D=2$ is also depicted.

In the present manuscript, we attempt to evaluate the correlation (or coherence) length of streaky structures using SPOD. It is also possible to use the cross-spectral density to evaluate lengthscales, similar to what is done by Jaunet et al. (2017); however, this requires taking specific choices for streamwise and radial positions, and a complete analysis for all possible positions becomes cumbersome. The use of SPOD circumvent these problems, as the dominant spatial structure in the whole cross-spectral density field is obtained in the leading eigenfunction. This allows a global estimate of lengthscales of dominant turbulent structures.

\subsection{Comparison with linear models}

We now focus on analysis of the radial shapes of the most energetic disturbances in the flow for $S t=0$ and azimuthal wavenumbers $m=3, m=7$ and $m=10$ at $x / D=2$, a region where linear mechanisms are expected to be more relevant due to the higher shear. Cross-sections of the leading SPOD modes are shown in figure 10(a,c,e) and, for these azimuthal wavenumbers, it can be seen that most of mode dynamics is concentrated in the sheared region, especially around the lipline, with low amplitudes in the central region where the mean velocity is approximately uniform. As previously mentioned, the shapes of the SPOD modes are similar to what is found for turbulent pipe flow (Hellström et al. 2015, 2016); the main difference in the present flow is the absence of the wall, showing that this is not a sine qua non condition for the appearance of streaky structures in a flow and that the sheared mean flow of the jet alone supports their emergence, consistent with the analysis of Ellingsen \& Palm (1975). Another conclusion is that the radial decay of the structures is also fast. However, one should be careful in the analysis of the radial decay of the structures; since the PIV window covered the interval $0<r / D<0.8$, structures with a radial support larger than that would be suppressed. Further analysis of the SPOD modes will be performed in the following sections, where a comparison between the most energetic structures with results from other tools (mostly used in wallbounded turbulence analysis) will be performed. All analyses were performed using the experimental Reynolds number, considering the molecular viscosity. 


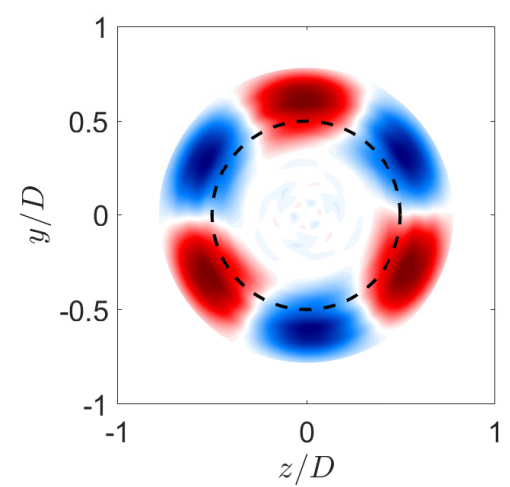

(a) First SPOD mode; $m=3$

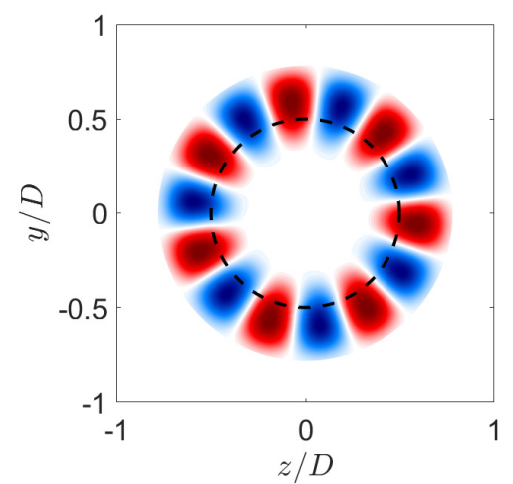

(c) First SPOD mode; $m=7$

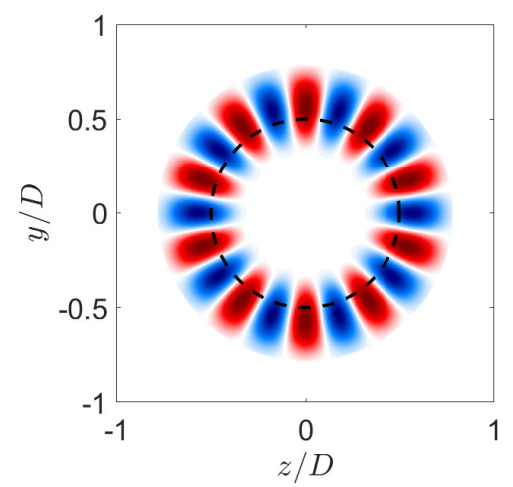

(e) First SPOD mode; $m=10$

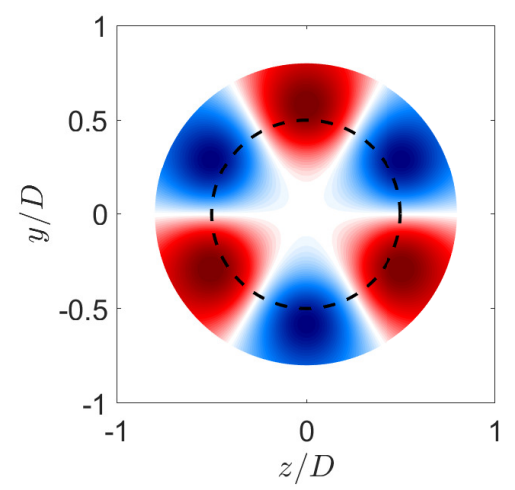

(b) First resolvent mode; $m=3$

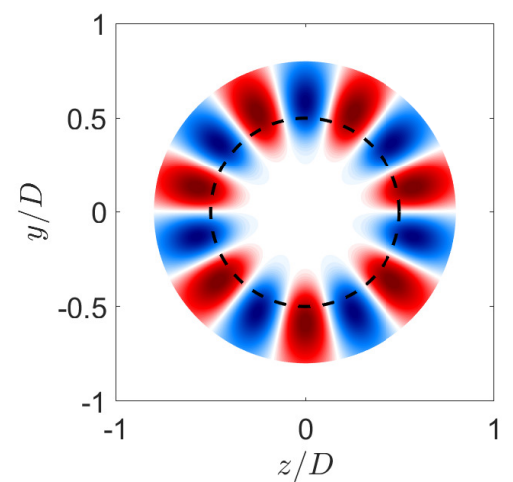

(d) First resolvent mode; $m=7$

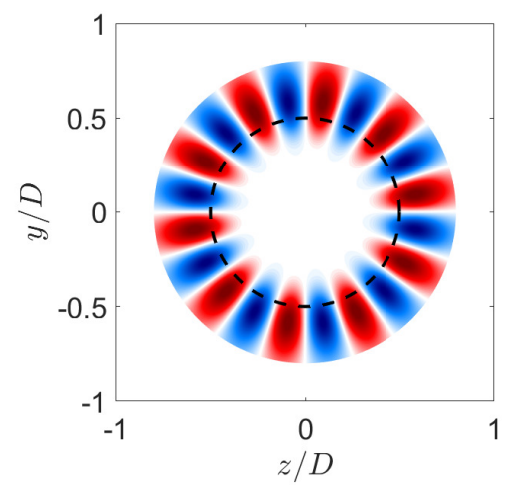

(f) First resolvent mode; $m=10$

FiguRE 10. First SPOD and resolvent modes for azimuthal wavenumbers $m=3, m=7$ and $m=10$ (real part). Optimal responses from resolvent analysis are shown for the same radial interval of the experiments for comparison $(r / D<0.8)$. The dashed lines indicates the position of the lipline. 


\subsubsection{Resolvent analysis}

Using the formulation described in section 3.2 , we obtain resolvent modes for the same frequency $\omega=0$ and wavenumbers $m=3 ; 7 ; 10$ for the $M=0.4$ jet at $x / D=2$. The structures of the first mode can be seen in figure $10(\mathrm{~b}, \mathrm{~d}, \mathrm{f})$. We note that these plots retain the key characteristics of the SPOD modes, suggesting that these structures can be obtained via resolvent analysis of the turbulent mean. Comparing figures $10(\mathrm{~b}, \mathrm{~d}, \mathrm{f})$ and 10(a,c,e), we see a good agreement in terms of both the position of the peak for $m=7$ and 10, using the lipline as reference point, and the radial support of these modes. For $m=3$, however, only the position of peak amplitude is captured by the resolvent analysis, which resulted in modes with radial shape that differ in width from the SPOD results. One of the reasons for that is the axial structure of the mode: as seen in figure 7 , amplitudes of the leading $m=3$ SPOD mode at $x / D=2$ are rather low, and become more relevant only further downstream. The local resolvent analysis is expected to better match the SPOD results if these do not vary considerably in $x$, since in the local resolvent analysis it is assumed that $k_{x}=0$, which leads to constant amplitudes in $x$. Still, even with this assumption, the local resolvent analysis is, on the whole, able to predict the shapes of the leading SPOD modes, particularly for higher azimuthal wavenumbers.

Another conclusion can be drawn from this analysis. It was shown by Semeraro et al. (2016); Towne et al. (2017) that resolvent and SPOD modes should be identical if the non-linear terms in the Navier-Stokes system were white in space. With that in mind, the resemblance between SPOD and resolvent modes in the present work suggests that the non-linear terms at that position (considered as forcing herein), may be related to uncorrelated structures, that behave in the manner of white noise, at least for high azimuthal wavenumbers.

Information about the optimal forcing shape is also of interest. One should recall that, for wall-bounded flows, the growth of streaks via the lift-up effect plays an important role (Schmid \& Henningson 2012; Brandt 2014), and is one of the dominant phenomena in the flow dynamics. These streaks are forced by streamwise vortices (or rolls) in a particular manner: positive streaks are related to forcing that advects flow from faster to slower regions, with the opposite happening for negative streaks. This roll-streak dynamics is also suggested to be one of the building-blocks that allows turbulence to self-sustain, as shown in Waleffe (1995); Hamilton et al. (1995); Hwang \& Bengana (2016). The optimal forcing from the resolvent analysis, and that leads to the streaky responses in figure 10(b,d,f), is shown in Figure 11. The optimal forcing is seen to consist mainly of radial and azimuthal fluctuations; the streamwise component is negligible for the azimuthal wavenumbers studied here. We can see that the same lift-up mechanism occurs for the present case: whenever a positive streak occurs in the response mode, it is always related to the movement of fluid from fast to slow regions (or from the inner part of the jet to the outer region). The presence of these vortices in the forcing modes supports the hypothesis that the lift-up mechanism is also relevant for turbulent jets.

\subsubsection{Transient growth}

A transient growth study of Michalke's velocity profiles for jets and slowly diffusing jet flows was performed by Jiménez-González \& Brancher (2017). In the present work, we extend the analysis to the turbulent jet, considering as base flow the experimental mean flow at $x / D=2$, using an implementation similar to the resolvent analysis described in the previous section. We are thus not looking for initial disturbances in a laminar flow that will generate the largest growth, triggering transition, as in the cited work; we focus, rather, on turbulent structures with the largest transient growth in time, and which would then naturally emerge as large-scale structures. This amounts to an identification 

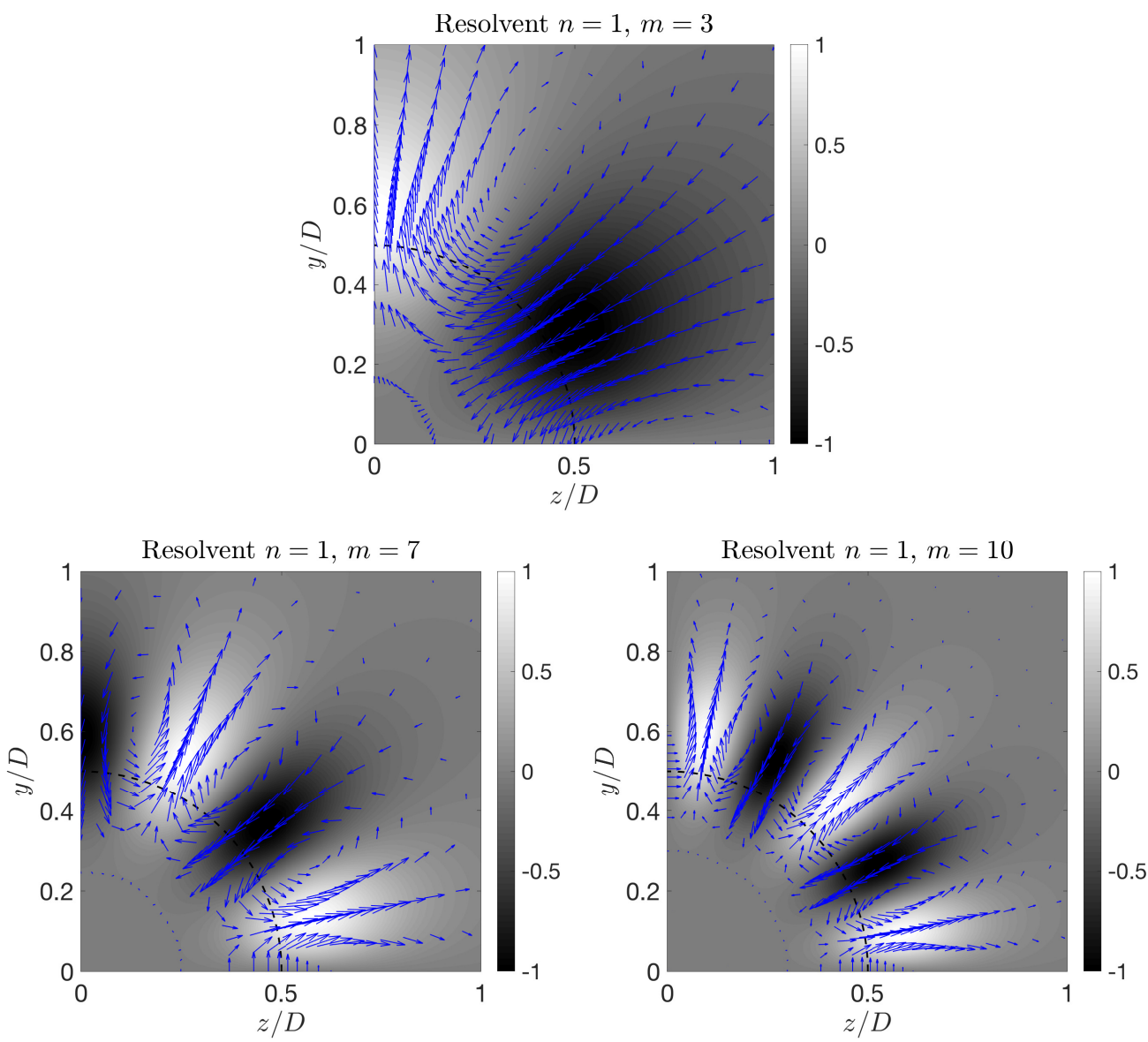

FIGURE 11. Radial and azimuthal components of the optimal forcing (arrows) and normalised streamwise component of optimal response (grayscale) from resolvent analysis for $m=3, m=7$ and $m=10$.

of the responses that predominate in the flow and the initial disturbances that lead to them. Also, using transient growth analysis, we obtain an idea of the time development of these disturbances, giving relevant information about the time scales over which this dynamics takes place.

The response at time of optimal growth for the transient growth analysis is similar to what was obtained by the resolvent analysis, with streaky structures concentrated close to the lipline. Figure 12 shows a comparison between the modes with optimal transient growth, those obtained by resolvent analysis and a cross-section of the global SPOD at $x / D=2$. These plots emphasize the conclusions drawn in the previous section: the transient growth analysis captures well the peak position of the streaks for the cases studied and even the radial support of the modes is well represented for higher azimuthal modes $(m=7 ; 10)$. It can also be seen that the structure of $m=3$ SPOD mode at this position has a much more experimental noise than in the other ones, which is probably due to its low relative amplitude at $x / D=2$. Still, the optimal responses from both resolvent and transient growth analyses match the SPOD modes for the azimuthal wavenumbers studied, with improving results as $\mathrm{m}$ increases.

One should recall that resolvent and SPOD modes were calculated for $\omega=0$, focusing 

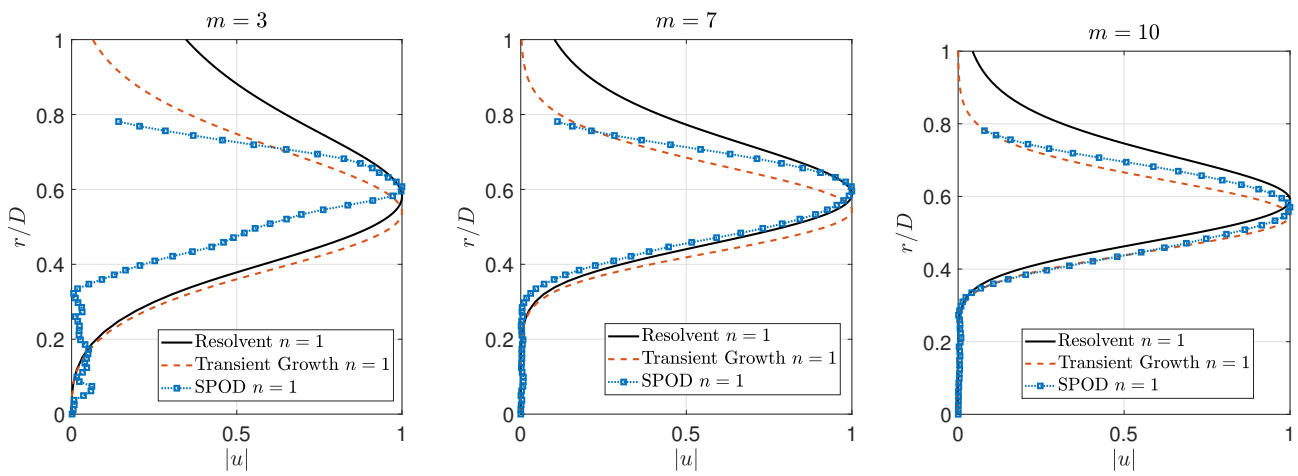

Figure 12. Comparison between SPOD modes $(\omega=0)$, response modes from resolvent analysis $\left(\omega=0, k_{x}=0\right)$ and response at the time of optimal growth from transient growth analysis $\left(k_{x}=0\right)$. The optimal is shown for $m=3, m=7$ and $m=10$.

on structures that have a very slow evolution (not to say invariant) in time. On the other hand, transient growth involves a different approach: the non-linear terms are set aside (there is no forcing) and we investigate initial disturbances that would have the highest growth for a given wavenumber. The fact that the present analysis shows a good agreement with resolvent modes is an indication that the dominant dynamics in this flow occurs in a low frequency, low wavenumber range, and is thus a long-time behaviour. This suggests the possibility of using non-modal stability methods for turbulent jets not only for the low-energy wavepackets, but also for the high-energy structures, similar to the approaches used for wall-bounded flows.

The slow dynamics of the phenomenon is supported by the growth curves for each case, depicted in figure 13(a). The large values of time for peak transient growth are also in accordance with the behaviour found by Jiménez-González \& Brancher (2017), who showed that the time of maximum growth scales with the Reynolds number; their results highlight a monotonic reduction of the optimal time of growth with increasing $m$, which also agrees with the trend identified in figure 13(a). One should nonetheless bear in mind that, since this analysis is restricted to a slice of the jet, this timescale is not related to a streamwise vortex generated close to the nozzle, which would evolve in time and space and become the resulting streak. In the locally parallel framework, the time is related to a vortex initially generated at the same position as the resulting streak, clouding the link between this result and the real time evolution of the structure.

Figure 13(b) shows how the growth curve changes with the axial station of the base flow chosen for the calculation for $m=7$, an azimuthal wavenumber whose first SPOD mode extends through the entire domain. This results shows that this linear mechanism is more relevant in regions closer to the nozzle, supporting our previous hypothesis. This behaviour is also similar to what was found by Jiménez-González \& Brancher (2017), who found smoother growth curves with lower optimal gains and higher optimal times for increasing shear layer momentum thickness; in our case, the momentum thickness of each axial section increases as we move downstream, leading to growth curves that have the same behaviour.

The initial disturbance that generates the optimally-growing streaks can be seen in figure 14. The similarities with results from Jiménez-González \& Brancher (2017) and with the optimal forcing from the resolvent analysis confirms that the streaks are generated via the lift-up mechanism. Initial perturbations in the shape of streamwise vortices are those that will suffer the largest growth, evolving into streamwise elongated 


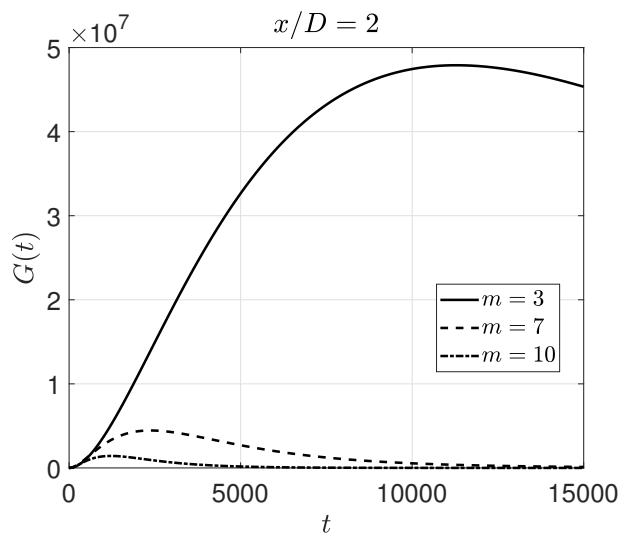

(a) $m=3 ; 7 ; 10$ and $x / D=2$

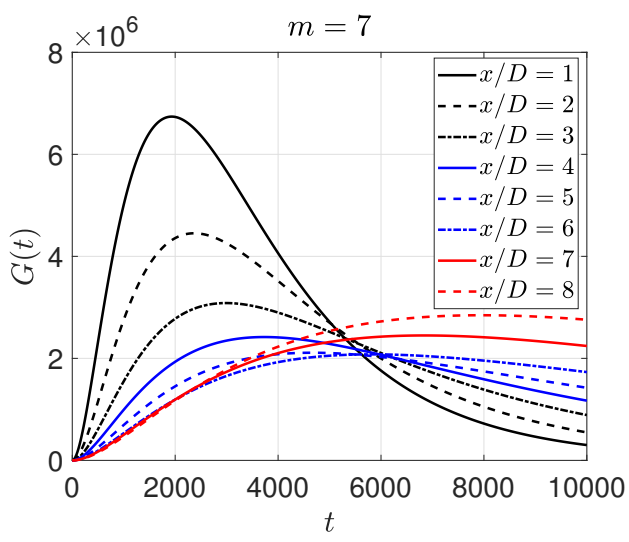

(b) $m=7$ and several streamwise positions

FiguRE 13. Growth curves from transient growth analysis $\left(k_{x}=0\right)$.

streaks, resembling those found by Cossu et al. (2009). These vortices are also related to those found by Tinney et al. (2008); the main novelty in the present study is their connection with the dominant streaks. Once again, the presence of these structures concentrated in the shear region, close to the lipline, is a suggestion that this, the lift-up mechanism, is related to any shear flow, not necessarily with the presence of a wall.

\subsubsection{Streaks downstream of the potential core}

In the previous sections we have chosen to perform the numerical analysis in regions closer to the nozzle, since the linear mechanisms we consider are expected to be stronger in this region due to the higher mean shear. Still, as shown in figure 6 and 7, these structures reach positions downstream of the potential core $(x / D>5.5$ for this jet), especially for low azimuthal wavenumbers; we here evaluate if linear models are able to predict streak shapes in downstream locations. Figure 15 shows the comparison between the radial dependency of the SPOD modes at $x / D=7$ for $m=3 ; 4 ; 5$ (wavenumbers for which the streaks have relevant amplitudes in this position) and the linear models using the base flow at this axial station, keeping the experimental Reynolds number in the analysis. At this position, the SPOD results show that the radial support of the most energetic structure considerably exceeds the measurement window, not only showing that streaks are larger in this region, but also making the analysis problematic, since about half of the radial information is lost. However, the models were able to qualitatively reproduce the sharp radial growth of the streak amplitude from the jet axis, with better results for transient growth analysis, especially for higher azimuthal wavenumbers.

\section{Conclusions}

In this work we have revisited the dual-plane, time-resolved, stereoscopic PIV database of a turbulent jet provided by Jaunet et al. (2017). We identify the presence of streamwise elongated, streaky structures in the turbulent field of a Mach 0.4 jet. To the best of our knowledge, this is the first time that turbulent streaks have been observed in turbulent jets. We have characterised the structures and highlighted similarities with those found in wall-bounded flows. The velocity fields (shown using Taylor hypothesis, as commonly done with experimental data of wall-bounded turbulence) exhibit a high resemblance with pipe flows and boundary layers, with the dominance of streaky structures, suggesting 
Large-scale, streaky structures in turbulent jets
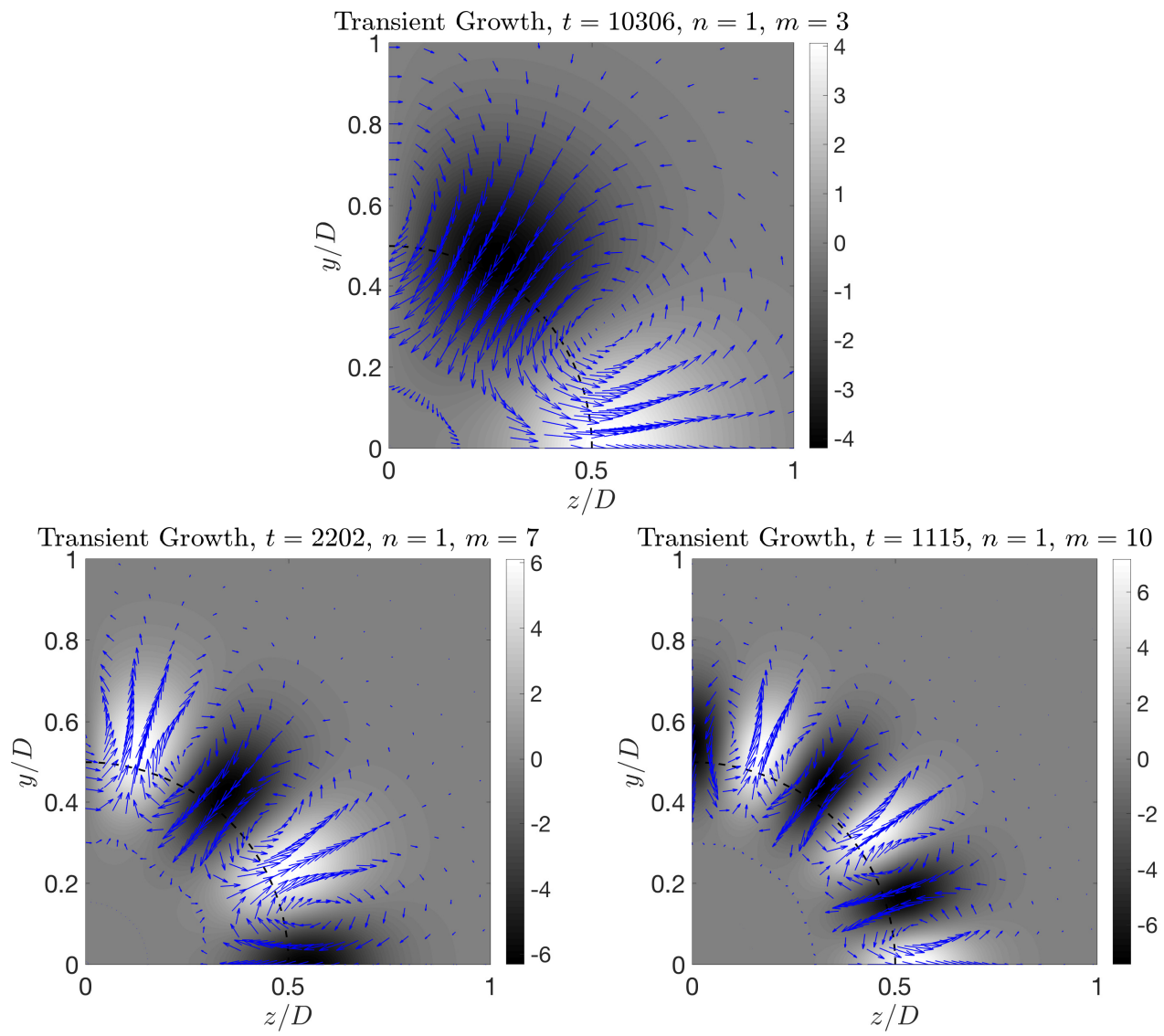

Figure 14. Radial and azimuthal components of the optimal initial disturbance (arrows) and streamwise component of optimal response (grayscale) from transient growth analysis for $m=3$, $m=7$ and $m=10$
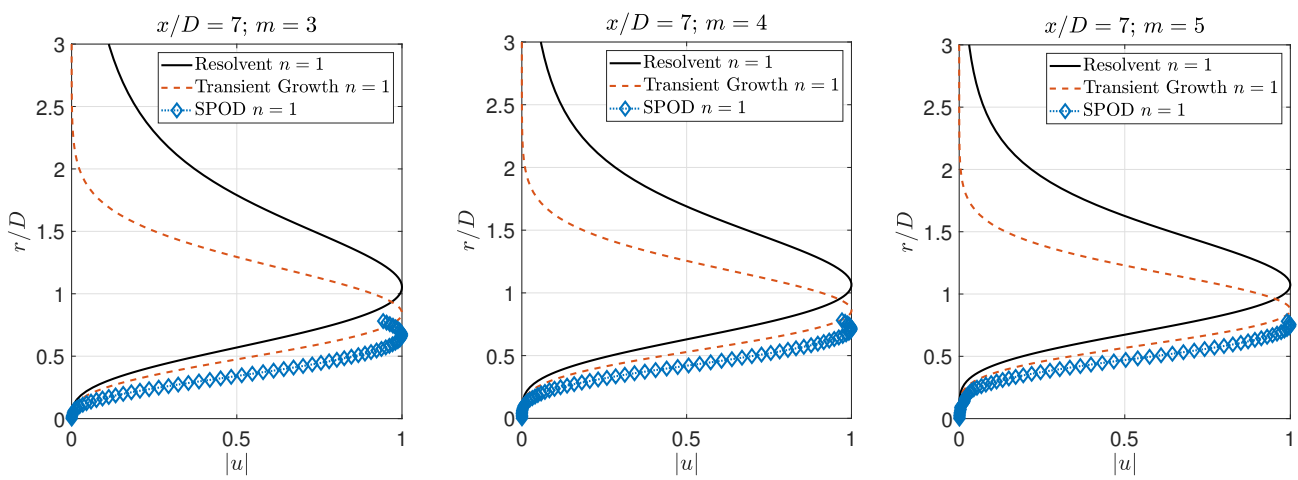

FiguRE 15. Comparison between SPOD modes $(\omega=0)$, response modes from resolvent analysis $\left(\omega=0, k_{x}=0\right)$ and response at the time of optimal growth from transient growth analysis $\left(k_{x}=0\right)$ for $x / D=7$. The optimal is shown for $m=3, m=4$ and $m=5$. 
that the underlying mechanism associated with such structures may be similar between jets and wall-bounded turbulence. Quantitative comparison of the energy spectrum with boundary layer experiments were also performed, showing again a similarity for the peak position of the spectrum. The growth of streaks in the axial direction was also inferred by the growth of the energy for $k_{x}=0$ as the PIV plane was moved downstream.

A spectral proper orthogonal decomposition of the streamwise velocity fluctuations clarified which modes are most relevant in the physical space of the experiment and how each mode was extended in the streamwise direction. Moreover, a scaling law, similar to that found by Hellström et al. (2016), was obtained, leading to a collapse of mode shapes for azimuthal wavenumbers $m \geqslant 3$, showing that these structures are also self-similar in the streamwise direction.

Cross-sections of the leading SPOD mode were compared with optimal flow responses from resolvent and transient growth analyses. Resolvent modes and SPOD modes are in agreement for the peak position and radial shape, with some discrepancies for radial positions $r / D>0.6$. The reasonable agreement obtained between the experimental SPOD and the resolvent indicates that the real forcing is likely to be a white noise in space, but improvement could be obtained if more realistic statistics of the non-linear terms were used. The shape of the optimal forcing and corresponding response relates the observed structures with the widely studied lift-up effect: for the turbulent jet, the forcing takes the form of streamwise vortices with velocities directed such that flow is moved from high- to low-speed regions, generating a positive streak; the opposite occurs for the appearance of a negative streak.

Even though transient growth analysis involves different assumptions, considering initial disturbances that lead to maximum growth in time, a good agreement was found for the optimal response and the SPOD modes. Results indicate structures of low frequency and wavenumber, characterised by a slow evolution in time. The optimal initial disturbance has a shape close to the optimal forcing from resolvent analysis, allowing a clearer connection with the lift-up effect studied in several other works, especially for wall-bounded flows (see Brandt (2014) and references therein).

Altogether, the results herein identify the presence of streaks in a high-Reynolds number, turbulent jet, displaying a direct connection with the lift-up effect. They show that this phenomenon is also present in natural unbounded flows such as jets, showing that the only element necessary for lift-up to occur is the presence of shear in a flow, meaning that the existence of a wall is not a sine qua non condition for the occurrence of streaks in turbulent flows. Moreover, since these coherent structures exist at the same time as the well-known Kelvin-Helmholtz wavepacket, they may be related to some of the features already studied in that framework. As observed by Liepmann \& Gharib (1992), axisymmetric Kelvin-Helmholtz vortices in transitional jets have secondary instabilities leading to the appearance of streamwise vortices, which may provide the required forcing leading to streaky structures. On the other hand, for transitional flows it was also observed that streaks have a stabilising effect on Kelvin-Helmholtz vortices (Marant \& Cossu 2018). From such studies, a promising direction for turbulent jets would be on a possible interplay between Kelvin-Helmholtz wavepackets, streamwise vortices and elongated streaks, since this could be responsible for wavepacket jitter and corresponding coherence decay (Cavalieri et al. 2011; Cavalieri \& Agarwal 2014).

Another interesting direction is the connection of the present findings with the dynamics of non-circular jets. In particular, nozzles serrations, or chevrons, are known to lead to streamwise vortices, which in turn modify the jet mean flow (Gutmark \& Grinstein 1999; Alkislar et al. 2007). This could be thought as the generation of steady streaks, which persist downstream, and tend to stabilise Kelvin-Helmholtz 
wavepackets (Lajús et al. 2015; Sinha et al. 2016; Marant \& Cossu 2018). Such steady streaky structures have been studied in turbulent boundary layers and related to the lift-up mechanism Pujals et al. (2010). The optimal forcings studied here could serve to guide nozzle design, increasing inhomogeneities of the shear layer in the azimuthal direction. Further work following this direction for turbulent jets is promising.

Acknowledgements: Petrônio Nogueira was funded by a CNPq scholarship. André Cavalieri acknowledges financial support by CNPq (grant number 310523/2017-6). We ackowledge professors Dan S. Henningson and Ardeshir Hanifi for the helpful insights in the discussions. We also thank professor Philipp Schlatter for providing results of large-eddy simulation of a turbulent boundary layer.

\section{Appendix A}

The underlying operators in section 3.2 were based on McKeon \& Sharma (2010)'s formulation and are defined below. The matrix $\mathscr{A}$, which accounts for the evolution of velocities is given by

$$
\mathscr{A}=\left[\begin{array}{ccc}
-\mathrm{i} k_{x} U+\frac{1}{R e}\left(\Delta+\frac{1}{r^{2}}\right) & -\frac{\partial U}{\partial r} & 0 \\
0 & -\mathrm{i} k_{x} U+\frac{1}{R e} \Delta & -\frac{1}{R e} \frac{2 \mathrm{i} m}{r^{2}} \\
0 & \frac{1}{R e} \frac{2 \mathrm{i} m}{r^{2}} & -\mathrm{i} k_{x} U+\frac{1}{R e} \Delta
\end{array}\right],
$$

where $\Delta=-k_{x}^{2}-\frac{\left(m^{2}+1\right)}{r^{2}}+\frac{\partial^{2}}{\partial r^{2}}+\frac{1}{r} \frac{\partial}{\partial r}$. In order to consider the pressure term in the formulation, the following operators are defined:

$$
\begin{array}{r}
\nabla_{C}=\left[\begin{array}{lll}
\mathrm{i} k_{x} & \frac{\partial}{\partial r}+\frac{1}{r} & \frac{\mathrm{i} m}{r}
\end{array}\right] \\
\nabla=\left[\begin{array}{c}
\mathrm{i} k_{x} \\
\frac{\partial}{\partial r} \\
\frac{\mathrm{i} m}{r}
\end{array}\right],
\end{array}
$$

which are responsible for both the inclusion of the pressure and the continuity equation. Finally, the operator $\mathscr{L}$ of the Linearised Navier-Stokes equations is defined as

$$
\mathscr{L}=\left[\begin{array}{cc}
\mathscr{A} & -\nabla \\
\nabla_{C} & 0
\end{array}\right]
$$

In order to isolate the time derivatives only in the velocity vector, we define the following matrix:

$$
\mathscr{H}=\left[\begin{array}{cc}
I_{3 \times 3} & 0_{3 \times 1} \\
0_{1 \times 3} & 0
\end{array}\right],
$$

where $I_{3 \times 3}$ is a $3 \times 3$ identity matrix and $0_{3 \times 1}, 0_{1 \times 3}$ are vectors of zeros (dimension $3 \times 1$ and $1 \times 3$, respectively). Similarly, we define matrix $\mathscr{B}$, which takes the forcing vector to a compact form:

$$
\mathscr{B}=\left[\begin{array}{l}
I_{3 \times 3} \\
0_{1 \times 3}
\end{array}\right]
$$


Finally, the filtering matrix that isolates only the streamwise velocity for the resolvent analysis is defined as:

$$
\mathscr{C}=\left[\begin{array}{cc}
I & 0 \\
0 & 0_{3 \times 3}
\end{array}\right]
$$

The boundary conditions were implemented as in Lesshafft \& Huerre (2007) and are dependent on the azimuthal mode as following:

$$
\begin{aligned}
& \left.\begin{array}{l}
u^{\prime}(0)=0 \\
v(0)=0 \\
w(0)=0 \\
p^{\prime}(0)=-\frac{2}{R e} v^{\prime \prime}(0)
\end{array}\right\} \text { for } m=0 \\
& \left.\begin{array}{l}
u(0)=0 \\
v^{\prime}(0)=0 \\
w(0) \pm \mathrm{i} v(0)=0 \\
p(0)=0
\end{array}\right\} \quad \text { for } \quad m= \pm 1 \\
& \left.\begin{array}{l}
u(0)=0 \\
v(0)=0 \\
w(0)=0 \\
p(0)=0
\end{array}\right\} \text { for } \quad|m|>1
\end{aligned}
$$

\section{REFERENCES}

Alkislar, Mehmet B, Krothapalli, Anjaneyulu \& Butler, GW 2007 The effect of streamwise vortices on the aeroacoustics of a mach 0.9 jet. Journal of Fluid Mechanics 578, 139-169.

Bernal, LP \& Roshko, A 1986 Streamwise vortex structure in plane mixing layers. Journal of Fluid Mechanics 170, 499-525.

Bogey, C., Marsden, O. \& Bailly, C. 2011 Large-eddy simulation of the flow and acoustic fields of a reynolds number 105 subsonic jet with tripped exit boundary layers. Physics of Fluids 23, 035104.

BRANDT, LUCA 2014 The lift-up effect: The linear mechanism behind transition and turbulence in shear flows. European Journal of Mechanics - B/Fluids 47, 80 - 96, enok Palm Memorial Volume.

Breakey, David E. S., Jordan, Peter, Cavalieri, André V. G., Nogueira, Petrônio A., LÉon, Olivier, Colonius, Tim \& Rodríguez, Daniel 2017 Experimental study of turbulent-jet wave packets and their acoustic efficiency. Phys. Rev. Fluids 2, 124601.

Butler, Kathryn M \& Farrell, Brian F 1992 Three-dimensional optimal perturbations in viscous shear flow. Physics of Fluids A: Fluid Dynamics 4 (8), 1637-1650.

Cavalieri, André, Jordan, Peter \& Lesshafft, Lutz 2019 Wave-packet models for jet dynamics and sound radiation. Applied Mechanics Reviews .

Cavalieri, André V. G. \& Agarwal, Anurag 2014 Coherence decay and its impact on sound radiation by wavepackets. Journal of Fluid Mechanics 748, 399-415.

Cavalieri, A. V. G., Jordan, P., Agarwal, A. \& Gervais, Y. 2011 Jittering wave-packet models for subsonic jet noise. Journal of Sound and Vibration 330 (18-19), 4474-4492.

Cavalieri, André V. G., Rodríguez, Daniel, Jordan, Peter, Colonius, Tim \& Gervais, Yves 2013 Wavepackets in the velocity field of turbulent jets. Journal of Fluid Mechanics 730, 559-592.

Cossu, Carlo, Pujals, Gregory \& Depardon, Sebastien 2009 Optimal transient growth and very large-scale structures in turbulent boundary layers. Journal of Fluid Mechanics 619, 79-94. 
Crighton, D. G. 1975 Basic principles of aerodynamic noise generation. Progress in Aerospace Sciences 16 (1), 31-96.

Crow, S. C. \& Champagne, F. H. 1971 Orderly structure in jet turbulence. Journal of Fluid Mechanics 48 (3), 547-591.

Del Alamo, Juan C \& Jimenez, Javier 2006 Linear energy amplification in turbulent channels. Journal of Fluid Mechanics 559, 205-213.

Dergham, G, Sipp, D \& Robinet, J-Ch 2013 Stochastic dynamics and model reduction of amplifier flows: the backward facing step flow. Journal of Fluid Mechanics 719, 406-430.

Eitel-Amor, Georg, Örlü, Ramis \& Schlatter, Philipp 2014 Simulation and validation of a spatially evolving turbulent boundary layer up to $\operatorname{re} \theta=8300$. International Journal of Heat and Fluid Flow 47, 57-69.

Ellingsen, T \& Palm, E 1975 Stability of linear flow. The Physics of Fluids 18 (4), 487-488.

Farrell, Brian F \& Ioannou, Petros J 2012 Dynamics of streamwise rolls and streaks in turbulent wall-bounded shear flow. Journal of Fluid Mechanics 708, 149-196.

GustavsSON, L HÅRKAN 1991 Energy growth of three-dimensional disturbances in plane poiseuille flow. Journal of Fluid Mechanics 224, 241-260.

Gutmark, EJ \& Grinstein, FF 1999 Flow control with noncircular jets. Annual review of fluid mechanics 31 (1), 239-272.

Hamilton, James M, Kim, John \& Waleffe, Fabian 1995 Regeneration mechanisms of near-wall turbulence structures. Journal of Fluid Mechanics 287, 317-348.

Hanifi, Ardeshir, Schmid, Peter J \& Henningson, Dan S 1996 Transient growth in compressible boundary layer flow. Physics of Fluids 8 (3), 826-837.

Hellström, Leo HO, Ganapathisubramani, Bharathram \& Smits, Alexander J 2015 The evolution of large-scale motions in turbulent pipe flow. Journal of Fluid Mechanics $\mathbf{7 7 9}, 701-715$.

Hellström, Leo HO, Marusic, Ivan \& Smits, Alexander J 2016 Self-similarity of the large-scale motions in turbulent pipe flow. Journal of Fluid Mechanics $\mathbf{7 9 2}$.

Hellström, Leo HO, Sinha, Aman \& Smits, Alexander J 2011 Visualizing the very-largescale motions in turbulent pipe flow. Physics of Fluids 23 (1), 011703.

Hultgren, Lennart S \& Gustavsson, L HÅkAn 1981 Algebraic growth of disturbances in a laminar boundary layer. The Physics of Fluids 24 (6), 1000-1004.

Hutchins, N \& MARUsic, Ivan 2007 Evidence of very long meandering features in the logarithmic region of turbulent boundary layers. Journal of Fluid Mechanics 579, 1-28.

Hwang, Yongyun \& Bengana, Yacine 2016 Self-sustaining process of minimal attached eddies in turbulent channel flow. Journal of Fluid Mechanics 795, 708-738.

Jaunet, Vincent, Jordan, Peter \& Cavalieri, André V 2017 Two-point coherence of wavepackets in turbulent jets. Physical Review Fluids in press.

JimÉnEZ-GonzÁLEZ, JI \& BRANCHER, P 2017 Transient energy growth of optimal streaks in parallel round jets. Physics of Fluids 29 (11), 114101.

Jordan, P. \& Colonius, T. 2013 Wave packets and turbulent jet noise. Annual Review of Fluid Mechanics 45 (1).

Jung, D., Gamard, S. \& George, W. K. 2004 Downstream evolution of the most energetic modes in a turbulent axisymmetric jet at high Reynolds number. Part 1. The near-field region. Journal of Fluid Mechanics 514, 173-204.

Kline, Stephen J, Reynolds, WC, Schraub, FA \& Runstadler, PW 1967 The structure of turbulent boundary layers. Journal of Fluid Mechanics 30 (4), 741-773.

Kozlov, VV, Grek, GR, Lofdahl, LL, Chernorai, VG \& Litvinenko, MV 2002 Role of localized streamwise structures in the process of transition to turbulence in boundary layers and jets. Journal of applied mechanics and technical physics 43 (2), 224-236.

Lajús, Francisco C, Cavalieri, André V \& Deschamps, Cesar J 2015 Spatial stability characteristics of non-circular jets. In 21st AIAA/CEAS Aeroacoustics Conference, p. 2537.

LANDAHL, MT 1980 A note on an algebraic instability of inviscid parallel shear flows. Journal of Fluid Mechanics 98 (2), 243-251.

Lesshafft, Lutz \& Huerre, Patrick 2007 Linear impulse response in hot round jets. Physics of Fluids 19 (2), 024102.

Lesshafft, Lutz, Semeraro, Onofrio, Jaunet, Vincent, Cavalieri, André VG \& 
JoRDAn, PETER 2018 Resolvent-based modelling of coherent wavepackets in a turbulent jet. arXiv preprint arXiv:1810.09340 .

Liepmann, Dorian \& GHarib, Morteza 1992 The role of streamwise vorticity in the near-field entrainment of round jets. Journal of Fluid Mechanics 245, 643-668.

Marant, Mathieu \& Cossu, Carlo 2018 Influence of optimally amplified streamwise streaks on the kelvin-helmholtz instability. Journal of Fluid Mechanics 838, 478-500.

Marusic, Ivan, BaArs, Woutijn J \& Hutchins, Nicholas 2017 Scaling of the streamwise turbulence intensity in the context of inner-outer interactions in wall turbulence. Physical Review Fluids 2 (10), 100502.

McKeon, BJ \& Sharma, AS 2010 A critical-layer framework for turbulent pipe flow. Journal of Fluid Mechanics 658, 336-382.

Mollo-Christensen, E. 1967 Jet noise and shear flow instability seen from an experimenter's viewpoint(Similarity laws for jet noise and shear flow instability as suggested by experiments). Journal of Applied Mechanics 34, 1-7.

Monty, JP, Stewart, JA, Williams, RC \& Chong, MS 2007 Large-scale features in turbulent pipe and channel flows. Journal of Fluid Mechanics 589, 147-156.

Pujals, Gregory, Cossu, Carlo \& Depardon, Sebastien 2010 Forcing large-scale coherent streaks in a zero-pressure-gradient turbulent boundary layer. Journal of Turbulence (11), N25.

Pujals, Gregory, García-Villalba, Manuel, Cossu, Carlo \& Depardon, Sebastien 2009 A note on optimal transient growth in turbulent channel flows. Physics of Fluids 21 (1), 015109.

SCARAnO, Fulvio 2001 Iterative image deformation methods in piv. Measurement science and technology 13 (1), R1.

Schmid, Peter J \& Henningson, Dan S 2012 Stability and transition in shear flows, , vol. 142. Springer Science \& Business Media.

Schoppa, W \& Hussain, FAzle 2002 Coherent structure generation in near-wall turbulence. Journal of fluid Mechanics 453, 57-108.

Semeraro, Onofrio, Jaunet, Vincent, Jordan, Peter, Cavalieri, André V \& LESSHAFFT, LUTZ 2016 Stochastic and harmonic optimal forcing in subsonic jets. In 22nd AIAA/CEAS Aeroacoustics Conference, p. 2935.

Sinha, Aniruddha, Gudmundsson, Kristuán, Xia, Hao \& Colonius, Tim 2016 Parabolized stability analysis of jets from serrated nozzles. Journal of Fluid Mechanics 789, 36-63.

Sinha, Aniruddha, Rodríguez, Daniel, Brès, Guillaume A. \& Colonius, Tim 2014 Wavepacket models for supersonic jet noise. Journal of Fluid Mechanics 742, 71-95.

Smith, CR \& Metzler, SP 1983 The characteristics of low-speed streaks in the near-wall region of a turbulent boundary layer. Journal of Fluid Mechanics 129, 27-54.

Smits, Alexander J, McKeon, Beverley J \& Marusic, Ivan 2011 High-reynolds number wall turbulence. Annual Review of Fluid Mechanics 43.

Tam, C. K. W. \& Burton, D. E. 1984 Sound generated by instability waves of supersonic flows. Part 2. Axisymmetric jets. Journal of Fluid Mechanics 138 (-1), 273-295.

Tinney, C. E., Glauser, M. N. \& Ukeiley, L. S. 2008 Low-dimensional characteristics of a transonic jet. part 1. proper orthogonal decomposition. Journal of Fluid Mechanics 612, 107.

Tissot, Gilles, Zhang, Mengqi, Lajús, Francisco C, Cavalieri, André VG \& Jordan, Peter 2017 Sensitivity of wavepackets in jets to nonlinear effects: the role of the critical layer. Journal of Fluid Mechanics 811, 95-137.

Tomkins, C. D. \& AdRiAn, R. J. 2005 Energetic spanwise modes in the logarithmic layer of a turbulent boundary layer. Journal of Fluid Mechanics 545, 141-162.

Towne, Aaron, Schmidt, Oliver T \& Colonius, Tim 2017 Spectral proper orthogonal decomposition and its relationship to dynamic mode decomposition and resolvent analysis. arXiv preprint arXiv:1708.04393 .

Towne, Aaron, Schmidt, Oliver T \& Colonius, Tim 2018 Spectral proper orthogonal decomposition and its relationship to dynamic mode decomposition and resolvent analysis. Journal of Fluid Mechanics 847, 821-867.

Trefethen, L. N. 2000 Spectral methods in MATLAB, , vol. 10. Society for Industrial Mathematics. 
WAleffe, FABian 1995 Hydrodynamic stability and turbulence: Beyond transients to a selfsustaining process. Studies in applied mathematics 95 (3), 319-343.

Westerweel, Jerry \& Scarano, Fulvio 2005 Universal outlier detection for piv data. Experiments in fluids 39 (6), 1096-1100. 